SUPPORTING INFORMATION

(22 pages)

\title{
Synthesis, Stereochemistry and Reactivity of Group 4 Metal Complexes That Contain a Chiral Tetradentate Diamine-Diamide Ligand
}

\author{
Jean-François Carpentier, ${ }^{\dagger}+$ Alfredo Martin,,+ Dale C. Swenson ${ }^{\ddagger}$ and Richard F. Jordan \\ Department of Chemistry, The University of Chicago, 5735 S. Ellis Ave., Chicago, IL 60637, USA \\ Department of Chemistry, The University of Iowa, Iowa City, IA 52242, USA
}

† Present address: UMR 6509 CNRS-Université de Rennes 1, Institut de Chimie, 35042 Rennes Cedex, France

† The University of Iowa

I The University of Chicago

* To whom correspondence should be addressed: Fax: (+1)773-702-0805; E-mail: rfjordan@uchicago.edu 


\section{Structural data for $C_{1}-\left(\mathrm{Me}_{2} \mathrm{PMEN}\right) \mathrm{ZrCl}_{2} \quad\left(C_{1}-3\right)$}

Table 1. Crystal data and structure refinement for $C_{1}-3$.

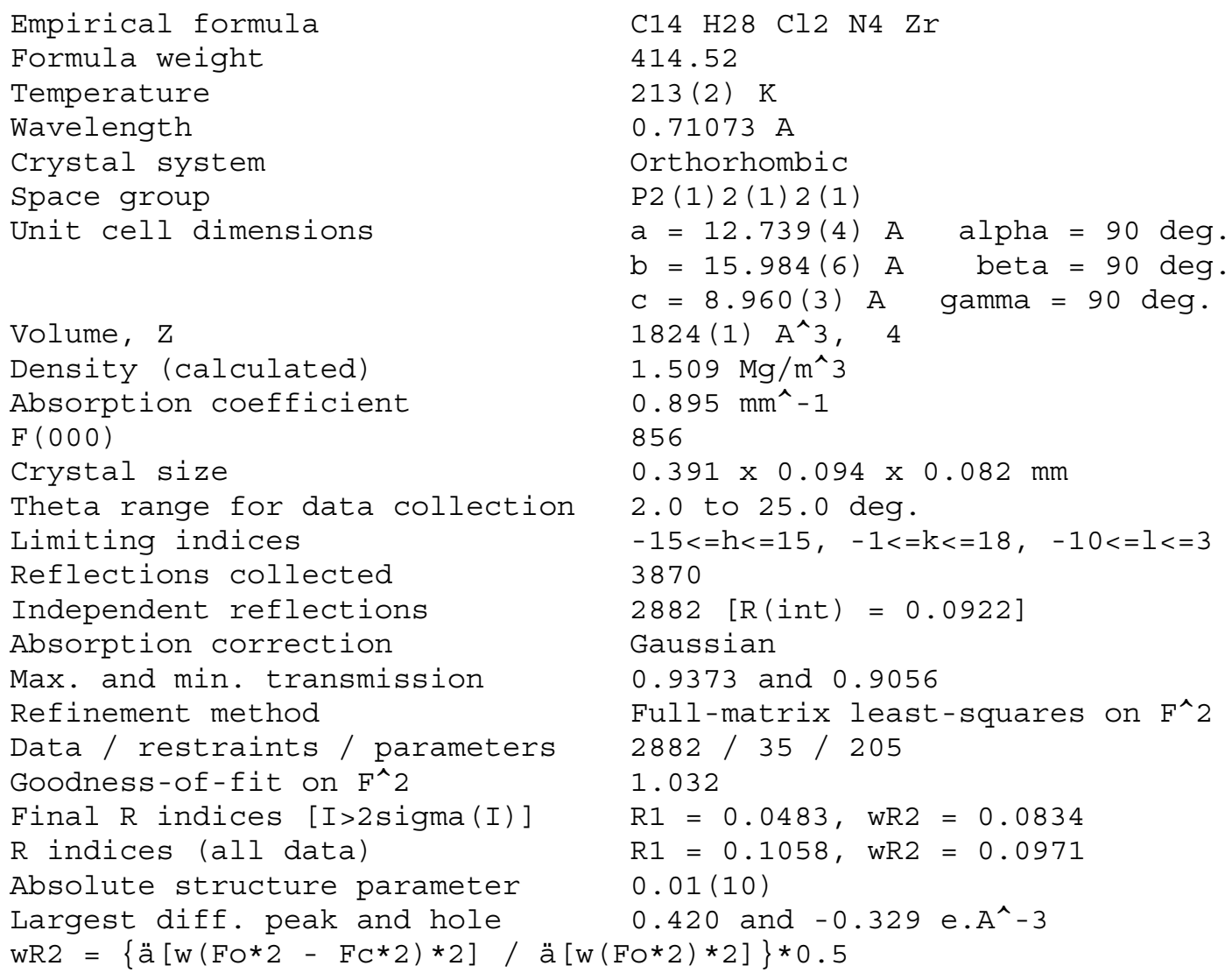


Table 2. Atomic coordinates $\left(x 10^{\wedge} 4\right)$ and equivalent isotropic displacement parameters $\left(\mathrm{A}^{\wedge} 2 \times 10^{\wedge} 3\right)$ for $C_{1}-3$. $\mathrm{U}(\mathrm{eq})$ is defined as one third of the trace of the orthogonalized Uij tensor.

\begin{tabular}{|c|c|c|c|c|}
\hline & $\mathrm{x}$ & $\mathrm{Y}$ & $\mathrm{z}$ & $\mathrm{U}$ ( eq) \\
\hline $\operatorname{Zr}(1)$ & $5449(1)$ & $3175(1)$ & $8029(1)$ & $29(1)$ \\
\hline $\mathrm{Cl}(1)$ & $6883(2)$ & $2146(1)$ & $7464(3)$ & $53(1)$ \\
\hline $\mathrm{Cl}(2)$ & $6304(2)$ & $4242(2)$ & $6475(3)$ & $60(1)$ \\
\hline $\mathrm{N}(1)$ & $3963(4)$ & $3645(4)$ & $7890(8)$ & $38(2)$ \\
\hline$C(2)$ & $3558(7)$ & $4441(6)$ & $8516(11)$ & $63(3)$ \\
\hline$C(3)$ & $2685(7)$ & $4747(6)$ & $7508(11)$ & $68(3)$ \\
\hline$C(4)$ & $2561(8)$ & $4098(6)$ & $6322(12)$ & $69(3)$ \\
\hline$C(5)$ & $3098(5)$ & $3321(5)$ & $6999(11)$ & $47(2)$ \\
\hline$C(6)$ & $3527(7)$ & $2706(6)$ & $5912(10)$ & $57(3)$ \\
\hline $\mathrm{N}(7)$ & $4379(5)$ & $2181(4)$ & $6579(7)$ & $37(2)$ \\
\hline$C(8)$ & $3940(7)$ & $1513(5)$ & $7582(10)$ & $56(3)$ \\
\hline$C(9)$ & $3807(6)$ & $1800(7)$ & $9174(9)$ & $53(2)$ \\
\hline $\mathrm{N}(10)$ & $4778(5)$ & $2168(4)$ & $9797(7)$ & $40(2)$ \\
\hline$C(11)$ & $4526(7)$ & $2697(6)$ & $11104(7)$ & $51(2)$ \\
\hline$C(12)$ & $5443(7)$ & $3267(5)$ & $11489(7)$ & $46(1)$ \\
\hline$C(13)$ & $5221(29)$ & $4048(12)$ & $12405(33)$ & $66(2)$ \\
\hline$C(14)$ & $6042(20)$ & $4713(13)$ & $12104(14)$ & $57(3)$ \\
\hline$C(15)$ & $6286(7)$ & $4449(5)$ & $10485(10)$ & $52(2)$ \\
\hline$C\left(13^{\prime}\right)$ & $5116(26)$ & $3943(9)$ & $12587(25)$ & $66(2)$ \\
\hline$C\left(14^{\prime}\right)$ & $5557(17)$ & $4701(10)$ & $11787(17)$ & $56(3)$ \\
\hline $\mathrm{N}(16)$ & $5817(4)$ & $3639(4)$ & $10082(7)$ & $36(1)$ \\
\hline$C(17)$ & $4912(6)$ & $1760(6)$ & $5302(9)$ & $56(2)$ \\
\hline$C(18)$ & $5501(8)$ & $1486(5)$ & $10264(10)$ & $59(2)$ \\
\hline
\end{tabular}


Table 3. Selected bond lengths [A] and angles [deg] for $C_{1}-3$.

\begin{tabular}{|c|c|}
\hline $\operatorname{Zr}(1)-\mathrm{N}(16)$ & $2.038(6)$ \\
\hline $\operatorname{Zr}(1)-N(1)$ & $2.039(6)$ \\
\hline $\operatorname{Zr}(1)-\mathrm{N}(10)$ & $2.415(6)$ \\
\hline $\operatorname{Zr}(1)-C l(2)$ & $2.457(2)$ \\
\hline $\operatorname{Zr}(1)-N(7)$ & $2.464(6)$ \\
\hline $\operatorname{Zr}(1)-C l(1)$ & $2.510(2)$ \\
\hline$N(1)-C(5)$ & $1.456(9)$ \\
\hline$N(1)-C(2)$ & $1.484(10)$ \\
\hline$C(2)-C(3)$ & $1.514(11)$ \\
\hline$C(3)-C(4)$ & $1.494(13)$ \\
\hline$C(4)-C(5)$ & $1.542(11)$ \\
\hline$C(5)-C(6)$ & $1.488(11)$ \\
\hline $\mathrm{C}(6)-\mathrm{N}(7)$ & $1.497(10)$ \\
\hline$N(7)-C(17)$ & $1.490(9)$ \\
\hline$N(7)-C(8)$ & $1.504(9)$ \\
\hline$C(8)-C(9)$ & $1.509(11)$ \\
\hline$C(9)-N(10)$ & $1.478(10)$ \\
\hline$N(10)-C(11)$ & $1.480(9)$ \\
\hline $\mathrm{N}(10)-\mathrm{C}(18)$ & $1.488(10)$ \\
\hline$C(11)-C(12)$ & $1.521(12)$ \\
\hline $\mathrm{C}(12)-\mathrm{N}(16)$ & $1.473(9)$ \\
\hline$C(12)-C(13)$ & $1.520(12)$ \\
\hline$C(12)-C\left(13^{\prime}\right)$ & $1.519(11)$ \\
\hline$C(13)-C(14)$ & $1.52(2)$ \\
\hline$C(14)-C(15)$ & $1.542(13)$ \\
\hline$C(15)-N(16)$ & $1.471(10)$ \\
\hline$C(15)-C\left(14{ }^{\prime}\right)$ & $1.545(13)$ \\
\hline$C\left(13^{\prime}\right)-C\left(14^{\prime}\right)$ & $1.52(2)$ \\
\hline $\mathrm{N}(16)-\operatorname{Zr}(1)-\mathrm{N}(1)$ & $97.7(2)$ \\
\hline $\mathrm{N}(16)-\operatorname{Zr}(1)-\mathrm{N}(10)$ & $74.5(2)$ \\
\hline$N(1)-\operatorname{Zr}(1)-N(10)$ & $87.5(2)$ \\
\hline $\mathrm{N}(16)-\operatorname{Zr}(1)-\mathrm{Cl}(2)$ & $99.0(2)$ \\
\hline$N(1)-\operatorname{Zr}(1)-C 1(2)$ & $96.9(2)$ \\
\hline$N(10)-\operatorname{Zr}(1)-C 1(2)$ & $172.6(2)$ \\
\hline $\mathrm{N}(16)-\operatorname{Zr}(1)-\mathrm{N}(7)$ & $147.0(2)$ \\
\hline$N(1)-\operatorname{Zr}(1)-N(7)$ & $72.1(2)$ \\
\hline$N(10)-\operatorname{Zr}(1)-N(7)$ & $73.8(2)$ \\
\hline $\mathrm{Cl}(2)-\operatorname{Zr}(1)-\mathrm{N}(7)$ & $113.2(2)$ \\
\hline $\mathrm{N}(16)-\operatorname{Zr}(1)-\mathrm{Cl}(1)$ & $104.7(2)$ \\
\hline $\mathrm{N}(1)-\operatorname{Zr}(1)-\mathrm{Cl}(1)$ & $154.7(2)$ \\
\hline $\mathrm{N}(10)-\operatorname{Zr}(1)-\mathrm{Cl}(1)$ & $87.3(2)$ \\
\hline $\mathrm{Cl}(2)-\operatorname{Zr}(1)-\mathrm{Cl}(1)$ & $91.04(9)$ \\
\hline$N(7)-\operatorname{Zr}(1)-C 1(1)$ & $82.7(2)$ \\
\hline$C(5)-N(1)-C(2)$ & $104.4(6)$ \\
\hline$C(5)-N(1)-\operatorname{Zr}(1)$ & $127.2(5)$ \\
\hline$C(2)-N(1)-\operatorname{Zr}(1)$ & $128.0(5)$ \\
\hline $\mathrm{N}(1)-\mathrm{C}(2)-\mathrm{C}(3)$ & $107.9(7)$ \\
\hline$C(4)-C(3)-C(2)$ & $106.1(7)$ \\
\hline$C(3)-C(4)-C(5)$ & $103.4(7)$ \\
\hline$N(1)-C(5)-C(6)$ & $108.4(6)$ \\
\hline$N(1)-C(5)-C(4)$ & $105.4(7)$ \\
\hline$C(6)-C(5)-C(4)$ & $115.9(8)$ \\
\hline$C(5)-C(6)-N(7)$ & $112.1(6)$ \\
\hline
\end{tabular}




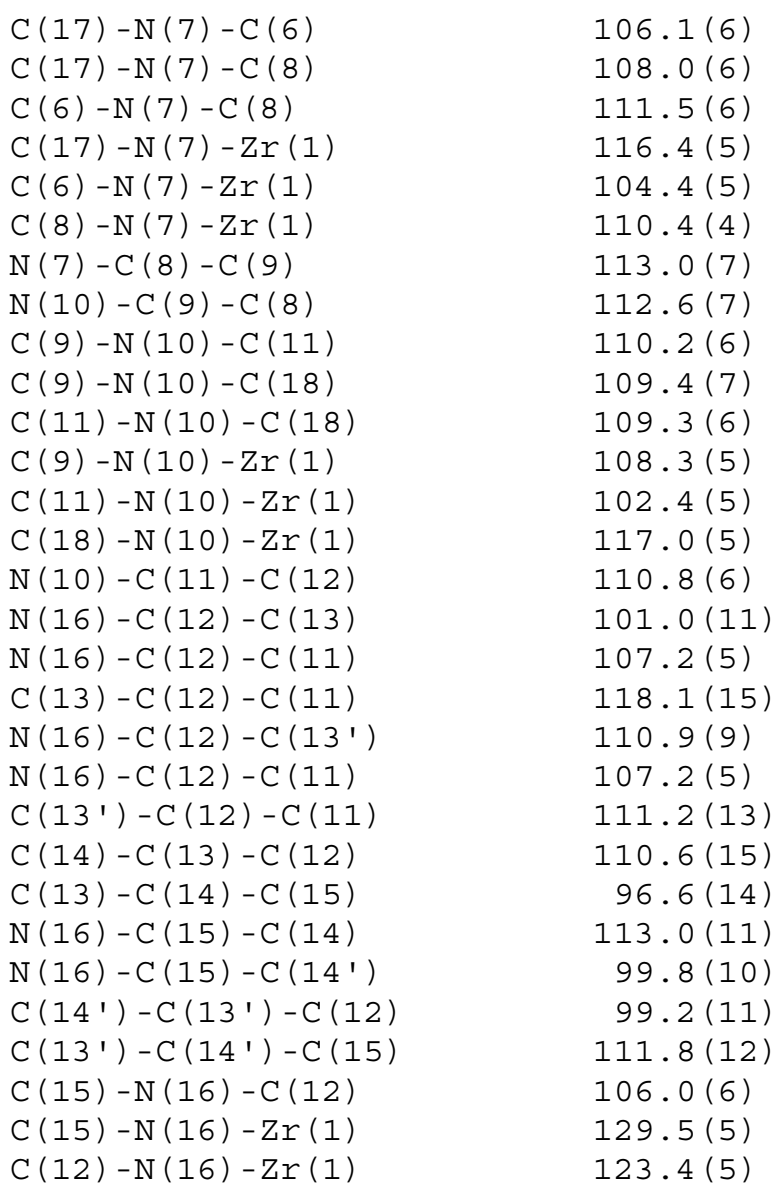

Symmetry transformations used to generate equivalent atoms: 
Table 4. Bond lengths [A] and angles [deg] for $C_{1}-3$.

\begin{tabular}{|c|c|}
\hline $\operatorname{Zr}(1)-N(16)$ & $2.038(6)$ \\
\hline $\operatorname{Zr}(1)-N(1)$ & $2.039(6)$ \\
\hline $\operatorname{Zr}(1)-N(10)$ & $2.415(6)$ \\
\hline $\operatorname{Zr}(1)-C l(2)$ & $2.457(2)$ \\
\hline $\operatorname{Zr}(1)-N(7)$ & $2.464(6)$ \\
\hline $\operatorname{Zr}(1)-C l(1)$ & $2.510(2)$ \\
\hline$N(1)-C(5)$ & $1.456(9)$ \\
\hline$N(1)-C(2)$ & $1.484(10)$ \\
\hline$C(2)-C(3)$ & $1.514(11)$ \\
\hline $\mathrm{C}(2)-\mathrm{H}(2 \mathrm{~A})$ & 0.97 \\
\hline $\mathrm{C}(2)-\mathrm{H}(2 \mathrm{~B})$ & 0.97 \\
\hline$C(3)-C(4)$ & $1.494(13)$ \\
\hline $\mathrm{C}(3)-\mathrm{H}(3 \mathrm{~A})$ & 0.97 \\
\hline $\mathrm{C}(3)-\mathrm{H}(3 \mathrm{~B})$ & 0.97 \\
\hline$C(4)-C(5)$ & $1.542(11)$ \\
\hline $\mathrm{C}(4)-\mathrm{H}(4 \mathrm{~A})$ & 0.97 \\
\hline $\mathrm{C}(4)-\mathrm{H}(4 \mathrm{~B})$ & 0.97 \\
\hline$C(5)-C(6)$ & $1.488(11)$ \\
\hline$C(5)-H(5)$ & 0.98 \\
\hline$C(6)-N(7)$ & $1.497(10)$ \\
\hline $\mathrm{C}(6)-\mathrm{H}(6 \mathrm{~A})$ & 0.97 \\
\hline $\mathrm{C}(6)-\mathrm{H}(6 \mathrm{~B})$ & 0.97 \\
\hline $\mathrm{N}(7)-\mathrm{C}(17)$ & $1.490(9)$ \\
\hline$N(7)-C(8)$ & $1.504(9)$ \\
\hline$C(8)-C(9)$ & $1.509(11)$ \\
\hline $\mathrm{C}(8)-\mathrm{H}(8 \mathrm{~A})$ & 0.97 \\
\hline $\mathrm{C}(8)-\mathrm{H}(8 \mathrm{~B})$ & 0.97 \\
\hline $\mathrm{C}(9)-\mathrm{N}(10)$ & $1.478(10)$ \\
\hline $\mathrm{C}(9)-\mathrm{H}(9 \mathrm{~A})$ & 0.97 \\
\hline $\mathrm{C}(9)-\mathrm{H}(9 \mathrm{~B})$ & 0.97 \\
\hline $\mathrm{N}(10)-\mathrm{C}(11)$ & $1.480(9)$ \\
\hline $\mathrm{N}(10)-\mathrm{C}(18)$ & $1.488(10)$ \\
\hline$C(11)-C(12)$ & $1.521(12)$ \\
\hline $\mathrm{C}(11)-\mathrm{H}(11 \mathrm{~A})$ & 0.97 \\
\hline $\mathrm{C}(11)-\mathrm{H}(11 \mathrm{~B})$ & 0.97 \\
\hline $\mathrm{C}(12)-\mathrm{N}(16)$ & $1.473(9)$ \\
\hline$C(12)-C(13)$ & $1.520(12)$ \\
\hline$C(12)-C\left(13^{\prime}\right)$ & $1.519(11)$ \\
\hline $\mathrm{C}(12)-\mathrm{H}(12)$ & 0.98 \\
\hline $\mathrm{C}(12)-\mathrm{H}\left(12^{\prime}\right)$ & 0.98 \\
\hline$C(13)-C(14)$ & $1.52(2)$ \\
\hline $\mathrm{C}(13)-\mathrm{H}(13 \mathrm{~A})$ & 0.97 \\
\hline$C(13)-H(13 B)$ & 0.97 \\
\hline$C(14)-C(15)$ & $1.542(13)$ \\
\hline $\mathrm{C}(14)-\mathrm{H}(14 \mathrm{~A})$ & 0.97 \\
\hline $\mathrm{C}(14)-\mathrm{H}(14 \mathrm{~B})$ & 0.97 \\
\hline$C(15)-N(16)$ & $1.471(10)$ \\
\hline$C(15)-C\left(14^{\prime}\right)$ & $1.545(13)$ \\
\hline $\mathrm{C}(15)-\mathrm{H}(15 \mathrm{~A})$ & 0.97 \\
\hline$C(15)-H(15 B)$ & 0.97 \\
\hline $\mathrm{C}(15)-\mathrm{H}(15 \mathrm{C})$ & 0.97 \\
\hline $\mathrm{C}(15)-\mathrm{H}(15 \mathrm{D})$ & 0.97 \\
\hline$C\left(13^{\prime}\right)-C\left(14^{\prime}\right)$ & $1.52(2)$ \\
\hline $\mathrm{C}\left(13^{\prime}\right)-\mathrm{H}(13 \mathrm{C})$ & 0.97 \\
\hline
\end{tabular}




\begin{tabular}{|c|c|}
\hline $\mathrm{C}\left(13^{\prime}\right)-\mathrm{H}(13 \mathrm{D})$ & 0.97 \\
\hline $\mathrm{C}\left(14^{\prime}\right)-\mathrm{H}(14 \mathrm{C})$ & 0.97 \\
\hline $\mathrm{C}\left(14^{\prime}\right)-\mathrm{H}(14 \mathrm{D})$ & 0.97 \\
\hline $\mathrm{C}(17)-\mathrm{H}(17 \mathrm{~A})$ & 0.96 \\
\hline $\mathrm{C}(17)-\mathrm{H}(17 \mathrm{~B})$ & 0.96 \\
\hline $\mathrm{C}(17)-\mathrm{H}(17 \mathrm{C})$ & 0.96 \\
\hline $\mathrm{C}(18)-\mathrm{H}(18 \mathrm{~A})$ & 0.96 \\
\hline $\mathrm{C}(18)-\mathrm{H}(18 \mathrm{~B})$ & 0.96 \\
\hline $\mathrm{C}(18)-\mathrm{H}(18 \mathrm{C})$ & 0.96 \\
\hline $\mathrm{N}(16)-\operatorname{Zr}(1)-\mathrm{N}(1)$ & $97.7(2)$ \\
\hline $\mathrm{N}(16)-\mathrm{Zr}(1)-\mathrm{N}(10)$ & $74.5(2)$ \\
\hline $\mathrm{N}(1)-\operatorname{Zr}(1)-\mathrm{N}(10)$ & $87.5(2)$ \\
\hline $\mathrm{N}(16)-\operatorname{Zr}(1)-\mathrm{Cl}(2)$ & $99.0(2)$ \\
\hline $\mathrm{N}(1)-\operatorname{Zr}(1)-\mathrm{Cl}(2)$ & $96.9(2)$ \\
\hline $\mathrm{N}(10)-\operatorname{Zr}(1)-\mathrm{Cl}(2)$ & $172.6(2)$ \\
\hline$N(16)-\operatorname{Zr}(1)-N(7)$ & $147.0(2)$ \\
\hline $\mathrm{N}(1)-\operatorname{Zr}(1)-\mathrm{N}(7)$ & $72.1(2)$ \\
\hline $\mathrm{N}(10)-\operatorname{Zr}(1)-\mathrm{N}(7)$ & $73.8(2)$ \\
\hline $\mathrm{Cl}(2)-\mathrm{Zr}(1)-\mathrm{N}(7)$ & $113.2(2)$ \\
\hline $\mathrm{N}(16)-\operatorname{Zr}(1)-\mathrm{Cl}(1)$ & $104.7(2)$ \\
\hline $\mathrm{N}(1)-\operatorname{Zr}(1)-\mathrm{Cl}(1)$ & $154.7(2)$ \\
\hline $\mathrm{N}(10)-\operatorname{Zr}(1)-\mathrm{Cl}(1)$ & $87.3(2)$ \\
\hline $\mathrm{Cl}(2)-\operatorname{Zr}(1)-\mathrm{Cl}(1)$ & $91.04(9)$ \\
\hline $\mathrm{N}(7)-\operatorname{Zr}(1)-\mathrm{Cl}(1)$ & $82.7(2)$ \\
\hline$C(5)-N(1)-C(2)$ & $104.4(6)$ \\
\hline$C(5)-N(1)-\operatorname{Zr}(1)$ & $127.2(5)$ \\
\hline$C(2)-N(1)-\operatorname{Zr}(1)$ & $128.0(5)$ \\
\hline$N(1)-C(2)-C(3)$ & $107.9(7)$ \\
\hline $\mathrm{N}(1)-\mathrm{C}(2)-\mathrm{H}(2 \mathrm{~A})$ & $110.1(5)$ \\
\hline $\mathrm{C}(3)-\mathrm{C}(2)-\mathrm{H}(2 \mathrm{~A})$ & $110.1(6)$ \\
\hline $\mathrm{N}(1)-\mathrm{C}(2)-\mathrm{H}(2 \mathrm{~B})$ & $110.1(5)$ \\
\hline$C(3)-C(2)-H(2 B)$ & $110.1(6)$ \\
\hline $\mathrm{H}(2 \mathrm{~A})-\mathrm{C}(2)-\mathrm{H}(2 \mathrm{~B})$ & 108.4 \\
\hline$C(4)-C(3)-C(2)$ & $106.1(7)$ \\
\hline$C(4)-C(3)-H(3 A)$ & $110.5(6)$ \\
\hline$C(2)-C(3)-H(3 A)$ & $110.5(6)$ \\
\hline$C(4)-C(3)-H(3 B)$ & $110.5(6)$ \\
\hline $\mathrm{C}(2)-\mathrm{C}(3)-\mathrm{H}(3 \mathrm{~B})$ & $110.5(6)$ \\
\hline $\mathrm{H}(3 \mathrm{~A})-\mathrm{C}(3)-\mathrm{H}(3 \mathrm{~B})$ & 108.7 \\
\hline$C(3)-C(4)-C(5)$ & $103.4(7)$ \\
\hline$C(3)-C(4)-H(4 A)$ & $111.1(6)$ \\
\hline$C(5)-C(4)-H(4 A)$ & $111.1(5)$ \\
\hline$C(3)-C(4)-H(4 B)$ & $111.1(5)$ \\
\hline$C(5)-C(4)-H(4 B)$ & $111.1(5)$ \\
\hline $\mathrm{H}(4 \mathrm{~A})-\mathrm{C}(4)-\mathrm{H}(4 \mathrm{~B})$ & 109.0 \\
\hline$N(1)-C(5)-C(6)$ & $108.4(6)$ \\
\hline$N(1)-C(5)-C(4)$ & $105.4(7)$ \\
\hline$C(6)-C(5)-C(4)$ & $115.9(8)$ \\
\hline $\mathrm{N}(1)-\mathrm{C}(5)-\mathrm{H}(5)$ & $109.0(5)$ \\
\hline$C(6)-C(5)-H(5)$ & $109.0(5)$ \\
\hline $\mathrm{C}(4)-\mathrm{C}(5)-\mathrm{H}(5)$ & $109.0(5)$ \\
\hline$C(5)-C(6)-N(7)$ & $112.1(6)$ \\
\hline $\mathrm{C}(5)-\mathrm{C}(6)-\mathrm{H}(6 \mathrm{~A})$ & $109.2(5)$ \\
\hline$N(7)-C(6)-H(6 A)$ & $109.2(5)$ \\
\hline$C(5)-C(6)-H(6 B)$ & $109.2(4)$ \\
\hline $\mathrm{N}(7)-\mathrm{C}(6)-\mathrm{H}(6 \mathrm{~B})$ & $109.2(4)$ \\
\hline
\end{tabular}




\begin{tabular}{|c|c|}
\hline $\mathrm{H}(6 \mathrm{~A})-\mathrm{C}(6)-\mathrm{H}(6 \mathrm{~B})$ & 107.9 \\
\hline$C(17)-N(7)-C(6)$ & $106.1(6)$ \\
\hline$C(17)-N(7)-C(8)$ & $108.0(6)$ \\
\hline$C(6)-N(7)-C(8)$ & $111.5(6)$ \\
\hline$C(17)-N(7)-\operatorname{Zr}(1)$ & $116.4(5)$ \\
\hline$C(6)-N(7)-\operatorname{Zr}(1)$ & $104.4(5)$ \\
\hline $\mathrm{C}(8)-\mathrm{N}(7)-\operatorname{Zr}(1)$ & $110.4(4)$ \\
\hline$N(7)-C(8)-C(9)$ & $113.0(7)$ \\
\hline $\mathrm{N}(7)-\mathrm{C}(8)-\mathrm{H}(8 \mathrm{~A})$ & $109.0(4)$ \\
\hline $\mathrm{C}(9)-\mathrm{C}(8)-\mathrm{H}(8 \mathrm{~A})$ & $109.0(5)$ \\
\hline $\mathrm{N}(7)-\mathrm{C}(8)-\mathrm{H}(8 \mathrm{~B})$ & $109.0(4)$ \\
\hline$C(9)-C(8)-H(8 B)$ & $109.0(5)$ \\
\hline $\mathrm{H}(8 \mathrm{~A})-\mathrm{C}(8)-\mathrm{H}(8 \mathrm{~B})$ & 107.8 \\
\hline$N(10)-C(9)-C(8)$ & $112.6(7)$ \\
\hline $\mathrm{N}(10)-\mathrm{C}(9)-\mathrm{H}(9 \mathrm{~A})$ & $109.1(5)$ \\
\hline$C(8)-C(9)-H(9 A)$ & $109.1(5)$ \\
\hline $\mathrm{N}(10)-\mathrm{C}(9)-\mathrm{H}(9 \mathrm{~B})$ & $109.1(4)$ \\
\hline$C(8)-C(9)-H(9 B)$ & $109.1(5)$ \\
\hline$H(9 A)-C(9)-H(9 B)$ & 107.8 \\
\hline$C(9)-N(10)-C(11)$ & $110.2(6)$ \\
\hline$C(9)-N(10)-C(18)$ & $109.4(7)$ \\
\hline $\mathrm{C}(11)-\mathrm{N}(10)-\mathrm{C}(18)$ & $109.3(6)$ \\
\hline$C(9)-N(10)-\operatorname{Zr}(1)$ & $108.3(5)$ \\
\hline $\mathrm{C}(11)-\mathrm{N}(10)-\operatorname{Zr}(1)$ & $102.4(5)$ \\
\hline$C(18)-N(10)-\operatorname{Zr}(1)$ & $117.0(5)$ \\
\hline $\mathrm{N}(10)-\mathrm{C}(11)-\mathrm{C}(12)$ & $110.8(6)$ \\
\hline $\mathrm{N}(10)-\mathrm{C}(11)-\mathrm{H}(11 \mathrm{~A})$ & $109.5(4)$ \\
\hline $\mathrm{C}(12)-\mathrm{C}(11)-\mathrm{H}(11 \mathrm{~A})$ & $109.5(4)$ \\
\hline $\mathrm{N}(10)-\mathrm{C}(11)-\mathrm{H}(11 \mathrm{~B})$ & $109.5(4)$ \\
\hline $\mathrm{C}(12)-\mathrm{C}(11)-\mathrm{H}(11 \mathrm{~B})$ & $109.5(5)$ \\
\hline $\mathrm{H}(11 \mathrm{~A})-\mathrm{C}(11)-\mathrm{H}(11 \mathrm{~B})$ & 108.1 \\
\hline$N(16)-C(12)-C(13)$ & $101.0(11)$ \\
\hline $\mathrm{N}(16)-\mathrm{C}(12)-\mathrm{C}(11)$ & $107.2(5)$ \\
\hline$C(13)-C(12)-C(11)$ & $118.1(15)$ \\
\hline $\mathrm{N}(16)-\mathrm{C}(12)-\mathrm{H}(12)$ & $110.0(4)$ \\
\hline $\mathrm{C}(13)-\mathrm{C}(12)-\mathrm{H}(12)$ & $110.0(17)$ \\
\hline $\mathrm{C}(11)-\mathrm{C}(12)-\mathrm{H}(12)$ & $110.0(5)$ \\
\hline$N(16)-C(12)-C\left(13^{\prime}\right)$ & $110.9(9)$ \\
\hline $\mathrm{N}(16)-\mathrm{C}(12)-\mathrm{C}(11)$ & $107.2(5)$ \\
\hline$C\left(13^{\prime}\right)-C(12)-C(11)$ & $111.2(13)$ \\
\hline $\mathrm{N}(16)-\mathrm{C}(12)-\mathrm{H}\left(12^{\prime}\right)$ & 109.1 \\
\hline $\mathrm{C}\left(13^{\prime}\right)-\mathrm{C}(12)-\mathrm{H}\left(12^{\prime}\right)$ & 109.2 \\
\hline $\mathrm{C}(11)-\mathrm{C}(12)-\mathrm{H}\left(12^{\prime}\right)$ & 109.1 \\
\hline$C(14)-C(13)-C(12)$ & $110.6(15)$ \\
\hline $\mathrm{C}(14)-\mathrm{C}(13)-\mathrm{H}(13 \mathrm{~A})$ & $109.5(18)$ \\
\hline $\mathrm{C}(12)-\mathrm{C}(13)-\mathrm{H}(13 \mathrm{~A})$ & $109.5(17)$ \\
\hline $\mathrm{C}(14)-\mathrm{C}(13)-\mathrm{H}(13 \mathrm{~B})$ & $109.6(15)$ \\
\hline$C(12)-C(13)-H(13 B)$ & $109.6(13)$ \\
\hline $\mathrm{H}(13 \mathrm{~A})-\mathrm{C}(13)-\mathrm{H}(13 \mathrm{~B})$ & 108.1 \\
\hline$C(13)-C(14)-C(15)$ & $96.6(14)$ \\
\hline $\mathrm{C}(13)-\mathrm{C}(14)-\mathrm{H}(14 \mathrm{~A})$ & $112.4(19)$ \\
\hline $\mathrm{C}(15)-\mathrm{C}(14)-\mathrm{H}(14 \mathrm{~A})$ & $112.4(10)$ \\
\hline $\mathrm{C}(13)-\mathrm{C}(14)-\mathrm{H}(14 \mathrm{~B})$ & $112.5(14)$ \\
\hline $\mathrm{C}(15)-\mathrm{C}(14)-\mathrm{H}(14 \mathrm{~B})$ & $112.4(9)$ \\
\hline $\mathrm{H}(14 \mathrm{~A})-\mathrm{C}(14)-\mathrm{H}(14 \mathrm{~B})$ & 110.0 \\
\hline$N(16)-C(15)-C(14)$ & $113.0(11)$ \\
\hline $\mathrm{N}(16)-\mathrm{C}(15)-\mathrm{H}(15 \mathrm{~A})$ & $109.0(4)$ \\
\hline
\end{tabular}




\begin{tabular}{|c|c|}
\hline $\mathrm{C}(14)-\mathrm{C}(15)-\mathrm{H}(15 \mathrm{~A})$ & $109.0(10)$ \\
\hline $\mathrm{N}(16)-\mathrm{C}(15)-\mathrm{H}(15 \mathrm{~B})$ & $109.0(4)$ \\
\hline$C(14)-C(15)-H(15 B)$ & $109.0(10)$ \\
\hline $\mathrm{H}(15 \mathrm{~A})-\mathrm{C}(15)-\mathrm{H}(15 \mathrm{~B})$ & 107.8 \\
\hline$N(16)-C(15)-C\left(14^{\prime}\right)$ & $99.8(10)$ \\
\hline $\mathrm{N}(16)-\mathrm{C}(15)-\mathrm{H}(15 \mathrm{C})$ & $111.8(9)$ \\
\hline $\mathrm{C}\left(14^{\prime}\right)-\mathrm{C}(15)-\mathrm{H}(15 \mathrm{C})$ & $111.8(10)$ \\
\hline $\mathrm{N}(16)-\mathrm{C}(15)-\mathrm{H}(15 \mathrm{D})$ & $111.8(8)$ \\
\hline $\mathrm{C}\left(14^{\prime}\right)-\mathrm{C}(15)-\mathrm{H}(15 \mathrm{D})$ & $111.8(12)$ \\
\hline $\mathrm{H}(15 \mathrm{C})-\mathrm{C}(15)-\mathrm{H}(15 \mathrm{D})$ & $109.5(11)$ \\
\hline$C\left(14^{\prime}\right)-C\left(13^{\prime}\right)-C(12)$ & $99.2(11)$ \\
\hline $\mathrm{C}\left(14^{\prime}\right)-\mathrm{C}\left(13^{\prime}\right)-\mathrm{H}(13 \mathrm{C})$ & $112.0(16)$ \\
\hline $\mathrm{C}(12)-\mathrm{C}\left(13^{\prime}\right)-\mathrm{H}(13 \mathrm{C})$ & $111.9(13)$ \\
\hline $\mathrm{C}\left(14^{\prime}\right)-\mathrm{C}\left(13^{\prime}\right)-\mathrm{H}(13 \mathrm{D})$ & $111.9(15)$ \\
\hline $\mathrm{C}(12)-\mathrm{C}\left(13^{\prime}\right)-\mathrm{H}(13 \mathrm{D})$ & $111.9(13)$ \\
\hline $\mathrm{H}(13 \mathrm{C})-\mathrm{C}\left(13^{\prime}\right)-\mathrm{H}(13 \mathrm{D})$ & 109.6 \\
\hline$C\left(13^{\prime}\right)-C\left(14^{\prime}\right)-C(15)$ & $111.8(12)$ \\
\hline $\mathrm{C}\left(13^{\prime}\right)-\mathrm{C}\left(14^{\prime}\right)-\mathrm{H}(14 \mathrm{C})$ & $109.3(14)$ \\
\hline $\mathrm{C}\left(15^{\prime}\right)-\mathrm{C}\left(14^{\prime}\right)-\mathrm{H}(14 \mathrm{C})$ & $109.3(9)$ \\
\hline $\mathrm{C}\left(13^{\prime}\right)-\mathrm{C}\left(14^{\prime}\right)-\mathrm{H}(14 \mathrm{D})$ & $109.2(17)$ \\
\hline $\mathrm{C}\left(15^{\prime}\right)-\mathrm{C}\left(14^{\prime}\right)-\mathrm{H}(14 \mathrm{D})$ & $109.3(9)$ \\
\hline $\mathrm{H}(14 \mathrm{C})-\mathrm{C}\left(14^{\prime}\right)-\mathrm{H}(14 \mathrm{D})$ & 107.9 \\
\hline$C(15)-N(16)-C(12)$ & $106.0(6)$ \\
\hline$C(15)-N(16)-\operatorname{Zr}(1)$ & $129.5(5)$ \\
\hline$C(12)-N(16)-\operatorname{Zr}(1)$ & $123.4(5)$ \\
\hline$N(7)-C(17)-H(17 A)$ & $109.5(4)$ \\
\hline $\mathrm{N}(7)-\mathrm{C}(17)-\mathrm{H}(17 \mathrm{~B})$ & $109.5(4)$ \\
\hline $\mathrm{H}(17 \mathrm{~A})-\mathrm{C}(17)-\mathrm{H}(17 \mathrm{~B})$ & 109.5 \\
\hline $\mathrm{N}(7)-\mathrm{C}(17)-\mathrm{H}(17 \mathrm{C})$ & $109.5(4)$ \\
\hline $\mathrm{H}(17 \mathrm{~A})-\mathrm{C}(17)-\mathrm{H}(17 \mathrm{C})$ & 109.5 \\
\hline $\mathrm{H}(17 \mathrm{~B})-\mathrm{C}(17)-\mathrm{H}(17 \mathrm{C})$ & 109.5 \\
\hline $\mathrm{N}(10)-\mathrm{C}(18)-\mathrm{H}(18 \mathrm{~A})$ & $109.5(4)$ \\
\hline $\mathrm{N}(10)-\mathrm{C}(18)-\mathrm{H}(18 \mathrm{~B})$ & $109.5(4)$ \\
\hline $\mathrm{H}(18 \mathrm{~A})-\mathrm{C}(18)-\mathrm{H}(18 \mathrm{~B})$ & 109.5 \\
\hline $\mathrm{N}(10)-\mathrm{C}(18)-\mathrm{H}(18 \mathrm{C})$ & $109.5(4)$ \\
\hline $\mathrm{H}(18 \mathrm{~A})-\mathrm{C}(18)-\mathrm{H}(18 \mathrm{C})$ & 109.5 \\
\hline $\mathrm{H}(18 \mathrm{~B})-\mathrm{C}(18)-\mathrm{H}(18 \mathrm{C})$ & 109.5 \\
\hline
\end{tabular}

Symmetry transformations used to generate equivalent atoms: 
Table 5. Anisotropic displacement parameters $\left(\mathrm{A}^{\wedge} 2 \times 10^{\wedge} 3\right)$ for $\mathrm{C}_{1}-3$. The anisotropic displacement factor exponent takes the form:

$-2 \mathrm{pi}^{\wedge} 2\left[\mathrm{~h}^{\wedge} 2 \mathrm{a}^{{ }^{\wedge} 2} \mathrm{U} 11+\ldots+2 \mathrm{~h} \mathrm{k} \mathrm{a}^{*} \mathrm{~b} \mathrm{~b}^{*} \mathrm{U} 12\right]$

\begin{tabular}{|c|c|c|c|c|c|c|}
\hline & U11 & U2 2 & U33 & U2 3 & U13 & U12 \\
\hline $\operatorname{Zr}(1)$ & $27(1)$ & $30(1)$ & $30(1)$ & $2(1)$ & $2(1)$ & $-1(1)$ \\
\hline $\mathrm{Cl}(1)$ & $39(1)$ & $56(1)$ & $64(2)$ & $-6(1)$ & $12(1)$ & $11(1)$ \\
\hline $\mathrm{Cl}(2)$ & $74(2)$ & $52(2)$ & $56(2)$ & $11(1)$ & $7(1)$ & $-25(1)$ \\
\hline $\mathrm{N}(1)$ & $41(3)$ & $31(3)$ & $41(4)$ & $-3(4)$ & $-17(3)$ & $5(3)$ \\
\hline$C(2)$ & $60(5)$ & $54(6)$ & $75(7)$ & $-28(6)$ & $-21(6)$ & $31(5)$ \\
\hline$C(3)$ & $65(5)$ & $47(6)$ & $93(9)$ & $-4(6)$ & $-23(5)$ & $28(5)$ \\
\hline$C(4)$ & $66(6)$ & $59(7)$ & $82(8)$ & $-5(6)$ & $-35(6)$ & $29(5)$ \\
\hline$C(5)$ & $42(3)$ & $44(5)$ & $53(4)$ & $-2(5)$ & $-12(5)$ & $7(3)$ \\
\hline$C(6)$ & $55(5)$ & $59(6)$ & $55(5)$ & $-7(5)$ & $-29(5)$ & $7(5)$ \\
\hline $\mathrm{N}(7)$ & $39(3)$ & $34(3)$ & $38(4)$ & $1(3)$ & $-1(3)$ & $3(3)$ \\
\hline$C(8)$ & $60(5)$ & $36(5)$ & $73(7)$ & $6(5)$ & $-1(5)$ & $-12(4)$ \\
\hline$C(9)$ & $46(4)$ & $55(6)$ & $59(5)$ & $13(6)$ & $11(4)$ & $-24(5)$ \\
\hline $\mathrm{N}(10)$ & $38(4)$ & $44(4)$ & $37(3)$ & $10(3)$ & $-4(3)$ & $0(3)$ \\
\hline$C(11)$ & $45(4)$ & $81(6)$ & $27(4)$ & $20(4)$ & $14(4)$ & $-1(6)$ \\
\hline$C(12)$ & $50(3)$ & $56(3)$ & $32(3)$ & $4(2)$ & $-8(3)$ & $11(3)$ \\
\hline$C(13)$ & $80(4)$ & $79(3)$ & $39(3)$ & $-16(3)$ & $-3(3)$ & $4(4)$ \\
\hline$C\left(13^{\prime}\right)$ & $80(4)$ & 79 (3) & $39(3)$ & $-16(3)$ & $-3(3)$ & $4(4)$ \\
\hline$C(14)$ & $61(8)$ & $63(5)$ & $47(5)$ & $-15(4)$ & $-26(7)$ & $16(5)$ \\
\hline$C\left(14^{\prime}\right)$ & $62(8)$ & $60(3)$ & $45(6)$ & $-16(4)$ & $-18(6)$ & $17(6)$ \\
\hline$C(15)$ & $58(5)$ & $43(4)$ & $54(4)$ & $-15(3)$ & $-8(3)$ & $1(3)$ \\
\hline $\mathrm{N}(16)$ & $33(3)$ & $38(3)$ & $38(2)$ & $0(2)$ & $-6(2)$ & $5(2)$ \\
\hline$C(17)$ & $70(5)$ & $46(5)$ & $53(5)$ & $-11(5)$ & $0(4)$ & $-2(5)$ \\
\hline$C(18)$ & $58(5)$ & $50(5)$ & $71(6)$ & $20(5)$ & $-2(6)$ & $9(6)$ \\
\hline
\end{tabular}


Table 6. Hydrogen coordinates $\left(\mathrm{x}^{\left.10^{\wedge} 4\right)}\right.$ ) and isotropic displacement parameters $\left(A^{\wedge} 2 \times 10^{\wedge} 3\right)$ for $C_{1}-3$.

\begin{tabular}{|c|c|c|c|c|}
\hline & $\mathrm{x}$ & $\mathrm{y}$ & z & $\mathrm{U}(\mathrm{eq})$ \\
\hline $\mathrm{H}(2 \mathrm{~A})$ & $4115(7)$ & $4853(6)$ & $8563(11)$ & 75 \\
\hline $\mathrm{H}(2 \mathrm{~B})$ & $3292(7)$ & $4351(6)$ & $9518(11)$ & 75 \\
\hline $\mathrm{H}(3 \mathrm{~A})$ & $2038(7)$ & $4811(6)$ & $8067(11)$ & 82 \\
\hline $\mathrm{H}(3 \mathrm{~B})$ & $2868(7)$ & $5283(6)$ & $7070(11)$ & 82 \\
\hline $\mathrm{H}(4 \mathrm{~A})$ & $2904(8)$ & $4269(6)$ & $5405(12)$ & 83 \\
\hline $\mathrm{H}(4 \mathrm{~B})$ & $1825(8)$ & $3990(6)$ & $6118(12)$ & 83 \\
\hline $\mathrm{H}(5)$ & $2600(5)$ & $3036(5)$ & 7659 (11) & 56 \\
\hline $\mathrm{H}(6 \mathrm{~A})$ & $3803(7)$ & $3004(6)$ & $5053(10)$ & 68 \\
\hline $\mathrm{H}(6 \mathrm{~B})$ & $2964(7)$ & $2346(6)$ & $5569(10)$ & 68 \\
\hline $\mathrm{H}(8 \mathrm{~A})$ & $4404(7)$ & $1032(5)$ & $7564(10)$ & 68 \\
\hline $\mathrm{H}(8 \mathrm{~B})$ & $3263(7)$ & $1337(5)$ & $7197(10)$ & 68 \\
\hline $\mathrm{H}(9 \mathrm{~A})$ & $3250(6)$ & $2213(7)$ & $9218(9)$ & 64 \\
\hline $\mathrm{H}(9 \mathrm{~B})$ & $3598(6)$ & $1328(7)$ & $9785(9)$ & 64 \\
\hline $\mathrm{H}(11 \mathrm{~A})$ & $4366(7)$ & $2343(6)$ & $11953(7)$ & 61 \\
\hline $\mathrm{H}(11 \mathrm{~B})$ & $3911(7)$ & $3034(6)$ & $10887(7)$ & 61 \\
\hline $\mathrm{H}(12)$ & $6007(7)$ & $2942(5)$ & $11955(7)$ & 55 \\
\hline $\mathrm{H}\left(12^{\prime}\right)$ & $6008(26)$ & $2933(9)$ & $11930(25)$ & 55 \\
\hline $\mathrm{H}(13 \mathrm{~A})$ & $4532(29)$ & $4264(12)$ & $12152(33)$ & 79 \\
\hline $\mathrm{H}(13 \mathrm{~B})$ & $5223(29)$ & $3908(12)$ & $13458(33)$ & 79 \\
\hline $\mathrm{H}(14 \mathrm{~A})$ & $6647(20)$ & $4661(13)$ & $12754(14)$ & 68 \\
\hline $\mathrm{H}(14 \mathrm{~B})$ & $5758(20)$ & $5275(13)$ & $12165(14)$ & 68 \\
\hline $\mathrm{H}(15 \mathrm{~A})$ & $6025(7)$ & $4875(5)$ & $9810(10)$ & 62 \\
\hline $\mathrm{H}(15 \mathrm{~B})$ & $7041(7)$ & $4416(5)$ & $10358(10)$ & 62 \\
\hline $\mathrm{H}(15 \mathrm{C})$ & $6251(4)$ & $4846(4)$ & $9668(7)$ & 62 \\
\hline $\mathrm{H}(15 \mathrm{D})$ & $7009(4)$ & $4389(4)$ & $10808(7)$ & 62 \\
\hline $\mathrm{H}(13 \mathrm{C})$ & $4359(26)$ & $3974(9)$ & $12693(25)$ & 79 \\
\hline $\mathrm{H}(13 \mathrm{D})$ & $5436(26)$ & $3864(9)$ & $13559(25)$ & 79 \\
\hline $\mathrm{H}(14 \mathrm{C})$ & $5946(17)$ & $5041(10)$ & 12492 (17) & 67 \\
\hline $\mathrm{H}(14 \mathrm{D})$ & $4982(17)$ & $5036(10)$ & $11403(17)$ & 67 \\
\hline $\mathrm{H}(17 \mathrm{~A})$ & $5389(37)$ & $1345(28)$ & $5674(9)$ & 85 \\
\hline $\mathrm{H}(17 \mathrm{~B})$ & $5293(40)$ & $2168(9)$ & $4731(42)$ & 85 \\
\hline $\mathrm{H}(17 \mathrm{C})$ & $4396(8)$ & $1497(33)$ & $4677(38)$ & 85 \\
\hline $\mathrm{H}(18 \mathrm{~A})$ & $5552(41)$ & $1078(21)$ & $9481(32)$ & 89 \\
\hline $\mathrm{H}(18 \mathrm{~B})$ & $5234(28)$ & $1224(27)$ & $11151(44)$ & 89 \\
\hline $\mathrm{H}(18 \mathrm{C})$ & $6183(16)$ & $1715(8)$ & $10463(70)$ & 89 \\
\hline
\end{tabular}


Structural data for $C_{2}-\left(\mathrm{Me}_{2} \mathrm{PMEN}\right) \mathrm{Ti}\left(\mathrm{CH}_{2} \mathrm{Ph}\right)_{2} \quad\left(C_{2}-6\right)$.

Table 1. Crystal data and structure refinement for $C_{2}-6$.

Empirical formula

Formula weight

Temperature

Wavelength

Crystal system

Space group

Unit cell dimensions

Volume, Z

Density (calculated)

Absorption coefficient

$\mathrm{F}(000)$

Crystal size

Theta range for data collection

Limiting indices

Reflections collected

Independent reflections

Absorption correction

Refinement method

Data / restraints / parameters

Goodness-of-fit on $\mathrm{F}^{\wedge} 2$

Final $R$ indices [I>2sigma(I)]

$R$ indices (all data)

Absolute structure parameter

Largest diff. peak and hole

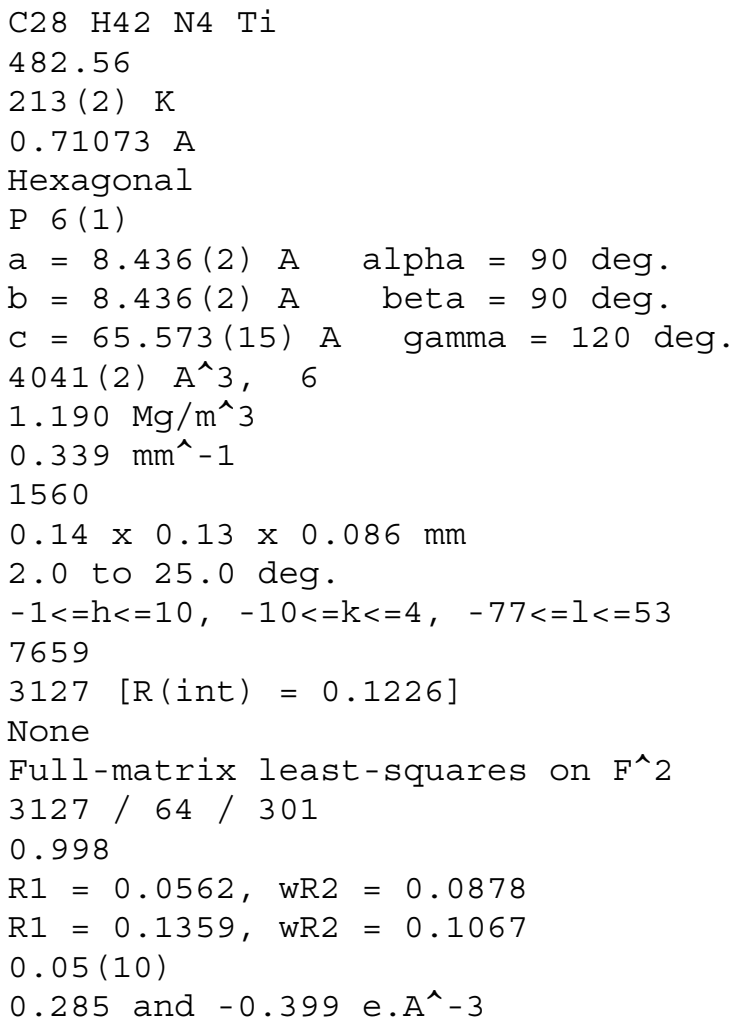


Table 2. Atomic coordinates $\left(x 10^{\wedge} 4\right)$ and equivalent isotropic displacement parameters $\left(A^{\wedge} 2 \times 10^{\wedge} 3\right)$ for $C_{2}-6$. U(eq) is defined as one third of the trace of the orthogonalized Uij tensor.

\begin{tabular}{|c|c|c|c|c|}
\hline & $\mathrm{x}$ & $\mathrm{Y}$ & z & $\mathrm{U}(\mathrm{eq})$ \\
\hline Ti (1) & $9286(2)$ & $4953(2)$ & $9556(1)$ & $43(1)$ \\
\hline $\mathrm{N}(1)$ & $10337(9)$ & $6275(8)$ & $9298(1)$ & $49(2)$ \\
\hline$C(2)$ & $12165(13)$ & $7318(14)$ & $9213(2)$ & $67(3)$ \\
\hline$C(3)$ & $12034(16)$ & 7069 (17) & $8986(2)$ & $100(4)$ \\
\hline$C(4)$ & $10031(17)$ & $6053(17)$ & $8938(2)$ & $95(3)$ \\
\hline$C(5)$ & $9185(15)$ & $6328(14)$ & $9130(2)$ & $74(3)$ \\
\hline$C(6)$ & 7267 (14) & $4913(16)$ & $9167(2)$ & $94(3)$ \\
\hline $\mathrm{N}(7)$ & $6822(9)$ & 4892 (11) & $9385(2)$ & $80(2)$ \\
\hline C (8) & $6829(15)$ & $6648(15)$ & $9444(2)$ & $110(3)$ \\
\hline C (9) & $7264(16)$ & $7092(15)$ & $9663(2)$ & $108(3)$ \\
\hline $\mathrm{N}(10)$ & $9067(13)$ & 7265 (11) & $9711(2)$ & $83(2)$ \\
\hline C (11) & 9205 (18) & $6982(16)$ & $9934(2)$ & $101(3)$ \\
\hline$C(12)$ & $7896(17)$ & $4952(16)$ & $9980(2)$ & $91(4)$ \\
\hline$C(13)$ & $8248(18)$ & $4216(15)$ & $10177(2)$ & $106(4)$ \\
\hline C (14) & $7253(17)$ & $2168(16)$ & $10135(2)$ & $111(4)$ \\
\hline$C(15)$ & $7218(14)$ & $2006(13)$ & $9905(2)$ & $78(3)$ \\
\hline$N(16)$ & $8054(10)$ & $3870(10)$ & $9813(1)$ & $61(2)$ \\
\hline C (17) & $5024(13)$ & $3280(13)$ & $9452(2)$ & $115(4)$ \\
\hline$C(18)$ & 10609 (15) & $9127(12)$ & $9652(2)$ & $99(4)$ \\
\hline C (19) & $12124(10)$ & $5955(11)$ & $9657(1)$ & $43(3)$ \\
\hline$C(20)$ & $12602(11)$ & $5563(12)$ & $9861(1)$ & $40(3)$ \\
\hline C (21) & $13520(15)$ & $6950(15)$ & $10005(2)$ & $76(3)$ \\
\hline$C(22)$ & $13955(18)$ & $6630(20)$ & $10198(2)$ & $105(5)$ \\
\hline$C(23)$ & $13474(18)$ & $4840(20)$ & $10249(2)$ & $105(5)$ \\
\hline C (24) & $12586(17)$ & $3509(19)$ & $10115(2)$ & $89(4)$ \\
\hline C (25) & $12152(14)$ & $3827(15)$ & $9918(2)$ & $64(4)$ \\
\hline$C(26)$ & $8473(11)$ & $2158(10)$ & $9464(1)$ & $49(3)$ \\
\hline C (27) & $8790(13)$ & $1643(12)$ & $9254(2)$ & $48(3)$ \\
\hline$C(28)$ & $10531(16)$ & $2270(14)$ & $9176(2)$ & $70(4)$ \\
\hline C (29) & $10855(16)$ & $1809(15)$ & $8980(2)$ & $80(4)$ \\
\hline$C(30)$ & $9420(20)$ & $673(17)$ & $8857(2)$ & $96(4)$ \\
\hline C (31) & $7703(17)$ & $43(16)$ & $8923(2)$ & $86(4)$ \\
\hline C (32) & $7410(15)$ & $517(14)$ & $9120(2)$ & $64(3)$ \\
\hline
\end{tabular}


Table 3. Selected bond lengths [A] and angles [deg] for $C_{2}-6$.

\begin{tabular}{|c|c|}
\hline $\mathrm{Ti}(1)-\mathrm{N}(16)$ & $1.952(8)$ \\
\hline $\mathrm{Ti}(1)-\mathrm{N}(1)$ & $1.979(7)$ \\
\hline $\mathrm{Ti}(1)-\mathrm{C}(26)$ & $2.185(8)$ \\
\hline $\mathrm{Ti}(1)-\mathrm{C}(19)$ & $2.204(8)$ \\
\hline $\mathrm{Ti}(1)-\mathrm{N}(10)$ & $2.287(8)$ \\
\hline $\mathrm{Ti}(1)-\mathrm{N}(7)$ & $2.342(7)$ \\
\hline$N(1)-C(2)$ & $1.451(10)$ \\
\hline$N(1)-C(5)$ & $1.482(11)$ \\
\hline$C(2)-C(3)$ & $1.499(12)$ \\
\hline$C(3)-C(4)$ & $1.497(14)$ \\
\hline$C(4)-C(5)$ & $1.522(12)$ \\
\hline$C(5)-C(6)$ & $1.473(14)$ \\
\hline $\mathrm{C}(6)-\mathrm{N}(7)$ & $1.475(12)$ \\
\hline$N(7)-C(17)$ & $1.510(11)$ \\
\hline$N(7)-C(8)$ & $1.529(12)$ \\
\hline$C(8)-C(9)$ & $1.488(13)$ \\
\hline $\mathrm{C}(9)-\mathrm{N}(10)$ & $1.488(13)$ \\
\hline $\mathrm{N}(10)-\mathrm{C}(11)$ & $1.495(13)$ \\
\hline $\mathrm{N}(10)-\mathrm{C}(18)$ & $1.506(12)$ \\
\hline$C(11)-C(12)$ & $1.533(16)$ \\
\hline $\mathrm{C}(12)-\mathrm{N}(16)$ & $1.471(12)$ \\
\hline$C(12)-C(13)$ & $1.526(15)$ \\
\hline$C(13)-C(14)$ & $1.522(14)$ \\
\hline$C(14)-C(15)$ & $1.511(12)$ \\
\hline $\mathrm{C}(15)-\mathrm{N}(16)$ & $1.491(10)$ \\
\hline$C(19)-C(20)$ & $1.485(10)$ \\
\hline$C(20)-C(25)$ & $1.367(12)$ \\
\hline$C(20)-C(21)$ & $1.396(13)$ \\
\hline$C(21)-C(22)$ & $1.381(14)$ \\
\hline$C(22)-C(23)$ & $1.395(18)$ \\
\hline$C(23)-C(24)$ & $1.326(16)$ \\
\hline$C(24)-C(25)$ & $1.406(14)$ \\
\hline$C(26)-C(27)$ & $1.510(11)$ \\
\hline$C(27)-C(28)$ & $1.386(13)$ \\
\hline$C(27)-C(32)$ & $1.388(13)$ \\
\hline$C(28)-C(29)$ & $1.408(13)$ \\
\hline$C(29)-C(30)$ & $1.368(15)$ \\
\hline$C(30)-C(31)$ & $1.341(15)$ \\
\hline$C(31)-C(32)$ & $1.409(14)$ \\
\hline $\mathrm{N}(16)-\mathrm{Ti}(1)-\mathrm{N}(1)$ & $171.0(3)$ \\
\hline $\mathrm{N}(16)-\mathrm{Ti}(1)-\mathrm{C}(26)$ & $85.8(3)$ \\
\hline $\mathrm{N}(1)-\mathrm{Ti}(1)-\mathrm{C}(26)$ & $100.5(3)$ \\
\hline $\mathrm{N}(16)-\mathrm{Ti}(1)-\mathrm{C}(19)$ & $98.2(3)$ \\
\hline $\mathrm{N}(1)-\mathrm{Ti}(1)-\mathrm{C}(19)$ & $86.9(3)$ \\
\hline $\mathrm{C}(26)-\mathrm{Ti}(1)-\mathrm{C}(19)$ & $101.0(3)$ \\
\hline $\mathrm{N}(16)-\mathrm{Ti}(1)-\mathrm{N}(10)$ & $76.3(3)$ \\
\hline $\mathrm{N}(1)-\mathrm{Ti}(1)-\mathrm{N}(10)$ & $96.0(3)$ \\
\hline $\mathrm{C}(26)-\mathrm{Ti}(1)-\mathrm{N}(10)$ & $157.9(4)$ \\
\hline $\mathrm{C}(19)-\mathrm{Ti}(1)-\mathrm{N}(10)$ & $94.3(4)$ \\
\hline $\mathrm{N}(16)-\mathrm{Ti}(1)-\mathrm{N}(7)$ & $98.4(3)$ \\
\hline $\mathrm{N}(1)-\mathrm{Ti}(1)-\mathrm{N}(7)$ & $75.1(3)$ \\
\hline $\mathrm{C}(26)-\mathrm{Ti}(1)-\mathrm{N}(7)$ & $92.7(3)$ \\
\hline $\mathrm{C}(19)-\mathrm{Ti}(1)-\mathrm{N}(7)$ & $159.1(3)$ \\
\hline
\end{tabular}




\begin{tabular}{|c|c|}
\hline $\mathrm{N}(10)-\mathrm{Ti}(1)-\mathrm{N}(7)$ & $77.5(3)$ \\
\hline$C(2)-N(1)-C(5)$ & $102.2(8)$ \\
\hline $\mathrm{C}(2)-\mathrm{N}(1)-\mathrm{Ti}(1)$ & $135.3(7)$ \\
\hline $\mathrm{C}(5)-\mathrm{N}(1)-\mathrm{Ti}(1)$ & $122.5(6)$ \\
\hline$N(1)-C(2)-C(3)$ & $108.3(9)$ \\
\hline$C(4)-C(3)-C(2)$ & $105.8(10)$ \\
\hline$C(3)-C(4)-C(5)$ & $102.7(10)$ \\
\hline$C(6)-C(5)-N(1)$ & $109.0(8)$ \\
\hline$C(6)-C(5)-C(4)$ & $114.9(11)$ \\
\hline$N(1)-C(5)-C(4)$ & $104.2(9)$ \\
\hline$C(5)-C(6)-N(7)$ & $109.8(10)$ \\
\hline$C(6)-N(7)-C(17)$ & $115.4(10)$ \\
\hline$C(6)-N(7)-C(8)$ & $110.9(9)$ \\
\hline$C(17)-N(7)-C(8)$ & $108.3(8)$ \\
\hline $\mathrm{C}(6)-\mathrm{N}(7)-\mathrm{Ti}(1)$ & $104.3(6)$ \\
\hline $\mathrm{C}(17)-\mathrm{N}(7)-\mathrm{Ti}(1)$ & $111.1(6)$ \\
\hline $\mathrm{C}(8)-\mathrm{N}(7)-\mathrm{Ti}(1)$ & $106.5(6)$ \\
\hline$C(9)-C(8)-N(7)$ & $111.8(10)$ \\
\hline$C(8)-C(9)-N(10)$ & $109.9(10)$ \\
\hline$C(9)-N(10)-C(11)$ & $110.4(9)$ \\
\hline$C(9)-N(10)-C(18)$ & $110.8(8)$ \\
\hline$C(11)-N(10)-C(18)$ & $108.8(10)$ \\
\hline $\mathrm{C}(9)-\mathrm{N}(10)-\mathrm{Ti}(1)$ & $109.7(7)$ \\
\hline $\mathrm{C}(11)-\mathrm{N}(10)-\mathrm{Ti}(1)$ & $104.7(6)$ \\
\hline $\mathrm{C}(18)-\mathrm{N}(10)-\mathrm{Ti}(1)$ & $112.3(6)$ \\
\hline$N(10)-C(11)-C(12)$ & $107.3(11)$ \\
\hline$N(16)-C(12)-C(13)$ & $106.3(9)$ \\
\hline$N(16)-C(12)-C(11)$ & $108.1(9)$ \\
\hline$C(13)-C(12)-C(11)$ & $116.2(13)$ \\
\hline$C(14)-C(13)-C(12)$ & $101.4(11)$ \\
\hline$C(15)-C(14)-C(13)$ & $105.0(9)$ \\
\hline$N(16)-C(15)-C(14)$ & $109.2(9)$ \\
\hline$C(12)-N(16)-C(15)$ & $102.3(9)$ \\
\hline $\mathrm{C}(12)-\mathrm{N}(16)-\mathrm{Ti}(1)$ & $122.9(7)$ \\
\hline $\mathrm{C}(15)-\mathrm{N}(16)-\mathrm{Ti}(1)$ & $134.6(7)$ \\
\hline $\mathrm{C}(20)-\mathrm{C}(19)-\mathrm{Ti}(1)$ & $123.3(6)$ \\
\hline$C(25)-C(20)-C(21)$ & $117.2(10)$ \\
\hline$C(25)-C(20)-C(19)$ & $121.4(9)$ \\
\hline$C(21)-C(20)-C(19)$ & $121.4(10)$ \\
\hline$C(22)-C(21)-C(20)$ & $123.1(11)$ \\
\hline$C(21)-C(22)-C(23)$ & $117.8(14)$ \\
\hline$C(24)-C(23)-C(22)$ & $119.7(14)$ \\
\hline$C(23)-C(24)-C(25)$ & $122.6(13)$ \\
\hline$C(20)-C(25)-C(24)$ & $119.5(11)$ \\
\hline $\mathrm{C}(27)-\mathrm{C}(26)-\mathrm{Ti}(1)$ & $123.8(6)$ \\
\hline$C(28)-C(27)-C(32)$ & $113.1(11)$ \\
\hline$C(28)-C(27)-C(26)$ & $122.3(10)$ \\
\hline$C(32)-C(27)-C(26)$ & $124.6(10)$ \\
\hline$C(27)-C(28)-C(29)$ & $123.2(11)$ \\
\hline$C(30)-C(29)-C(28)$ & $120.3(12)$ \\
\hline$C(31)-C(30)-C(29)$ & $119.4(14)$ \\
\hline$C(30)-C(31)-C(32)$ & $119.3(12)$ \\
\hline$C(27)-C(32)-C(31)$ & $124.7(11)$ \\
\hline
\end{tabular}

Symmetry transformations used to generate equivalent atoms: 
Table 4. Bond lengths [A] and angles [deg] for $C_{2}-6$.

\begin{tabular}{|c|c|}
\hline $\mathrm{Ti}(1)-\mathrm{N}(16)$ & $1.952(8)$ \\
\hline $\mathrm{Ti}(1)-\mathrm{N}(1)$ & $1.979(7)$ \\
\hline $\operatorname{Ti}(1)-C(26)$ & $2.185(8)$ \\
\hline $\operatorname{Ti}(1)-\mathrm{C}(19)$ & $2.204(8)$ \\
\hline $\operatorname{Ti}(1)-\mathrm{N}(10)$ & $2.287(8)$ \\
\hline Ti(1)-N(7) & $2.342(7)$ \\
\hline $\mathrm{N}(1)-\mathrm{C}(2)$ & $1.451(10)$ \\
\hline$N(1)-C(5)$ & $1.482(11)$ \\
\hline$C(2)-C(3)$ & $1.499(12)$ \\
\hline $\mathrm{C}(2)-\mathrm{H}(2 \mathrm{~A})$ & 0.9700 \\
\hline $\mathrm{C}(2)-\mathrm{H}(2 \mathrm{~B})$ & 0.9700 \\
\hline$C(3)-C(4)$ & $1.497(14)$ \\
\hline $\mathrm{C}(3)-\mathrm{H}(3 \mathrm{~A})$ & 0.9700 \\
\hline $\mathrm{C}(3)-\mathrm{H}(3 \mathrm{~B})$ & 0.9700 \\
\hline$C(4)-C(5)$ & $1.522(12)$ \\
\hline $\mathrm{C}(4)-\mathrm{H}(4 \mathrm{~A})$ & 0.9700 \\
\hline $\mathrm{C}(4)-\mathrm{H}(4 \mathrm{~B})$ & 0.9700 \\
\hline$C(5)-C(6)$ & $1.473(14)$ \\
\hline$C(5)-H(5)$ & 0.9800 \\
\hline$C(6)-N(7)$ & $1.475(12)$ \\
\hline $\mathrm{C}(6)-\mathrm{H}(6 \mathrm{~A})$ & 0.9700 \\
\hline $\mathrm{C}(6)-\mathrm{H}(6 \mathrm{~B})$ & 0.9700 \\
\hline$N(7)-C(17)$ & $1.510(11)$ \\
\hline$N(7)-C(8)$ & $1.529(12)$ \\
\hline$C(8)-C(9)$ & $1.488(13)$ \\
\hline $\mathrm{C}(8)-\mathrm{H}(8 \mathrm{~A})$ & 0.9700 \\
\hline $\mathrm{C}(8)-\mathrm{H}(8 \mathrm{~B})$ & 0.9700 \\
\hline$C(9)-N(10)$ & $1.488(13)$ \\
\hline $\mathrm{C}(9)-\mathrm{H}(9 \mathrm{~A})$ & 0.9700 \\
\hline $\mathrm{C}(9)-\mathrm{H}(9 \mathrm{~B})$ & 0.9700 \\
\hline $\mathrm{N}(10)-\mathrm{C}(11)$ & $1.495(13)$ \\
\hline $\mathrm{N}(10)-\mathrm{C}(18)$ & $1.506(12)$ \\
\hline$C(11)-C(12)$ & $1.533(16)$ \\
\hline $\mathrm{C}(11)-\mathrm{H}(11 \mathrm{~A})$ & 0.9700 \\
\hline $\mathrm{C}(11)-\mathrm{H}(11 \mathrm{~B})$ & 0.9700 \\
\hline $\mathrm{C}(12)-\mathrm{N}(16)$ & $1.471(12)$ \\
\hline$C(12)-C(13)$ & 1.526 (15) \\
\hline $\mathrm{C}(12)-\mathrm{H}(12)$ & 0.9800 \\
\hline$C(13)-C(14)$ & $1.522(14)$ \\
\hline $\mathrm{C}(13)-\mathrm{H}(13 \mathrm{~A})$ & 0.9700 \\
\hline $\mathrm{C}(13)-\mathrm{H}(13 \mathrm{~B})$ & 0.9700 \\
\hline$C(14)-C(15)$ & $1.511(12)$ \\
\hline $\mathrm{C}(14)-\mathrm{H}(14 \mathrm{~A})$ & 0.9700 \\
\hline $\mathrm{C}(14)-\mathrm{H}(14 \mathrm{~B})$ & 0.9700 \\
\hline$C(15)-N(16)$ & $1.491(10)$ \\
\hline $\mathrm{C}(15)-\mathrm{H}(15 \mathrm{~A})$ & 0.9700 \\
\hline $\mathrm{C}(15)-\mathrm{H}(15 \mathrm{~B})$ & 0.9700 \\
\hline $\mathrm{C}(17)-\mathrm{H}(17 \mathrm{~A})$ & 0.9600 \\
\hline $\mathrm{C}(17)-\mathrm{H}(17 \mathrm{~B})$ & 0.9600 \\
\hline $\mathrm{C}(17)-\mathrm{H}(17 \mathrm{C})$ & 0.9600 \\
\hline $\mathrm{C}(18)-\mathrm{H}(18 \mathrm{~A})$ & 0.9600 \\
\hline $\mathrm{C}(18)-\mathrm{H}(18 \mathrm{~B})$ & 0.9600 \\
\hline $\mathrm{C}(18)-\mathrm{H}(18 \mathrm{C})$ & 0.9600 \\
\hline$C(19)-C(20)$ & $1.485(10)$ \\
\hline
\end{tabular}




\begin{tabular}{|c|c|}
\hline $\mathrm{C}(19)-\mathrm{H}(19 \mathrm{~A})$ & 0.9700 \\
\hline $\mathrm{C}(19)-\mathrm{H}(19 \mathrm{~B})$ & 0.9700 \\
\hline$C(20)-C(25)$ & $1.367(12)$ \\
\hline$C(20)-C(21)$ & $1.396(13)$ \\
\hline$C(21)-C(22)$ & $1.381(14)$ \\
\hline $\mathrm{C}(21)-\mathrm{H}(21 \mathrm{~A})$ & 0.9300 \\
\hline$C(22)-C(23)$ & $1.395(18)$ \\
\hline $\mathrm{C}(22)-\mathrm{H}(22 \mathrm{~A})$ & 0.9300 \\
\hline$C(23)-C(24)$ & $1.326(16)$ \\
\hline $\mathrm{C}(23)-\mathrm{H}(23 \mathrm{~A})$ & 0.9300 \\
\hline$C(24)-C(25)$ & $1.406(14)$ \\
\hline $\mathrm{C}(24)-\mathrm{H}(24 \mathrm{~A})$ & 0.9300 \\
\hline $\mathrm{C}(25)-\mathrm{H}(25 \mathrm{~A})$ & 0.9300 \\
\hline$C(26)-C(27)$ & $1.510(11)$ \\
\hline $\mathrm{C}(26)-\mathrm{H}(26 \mathrm{~A})$ & 0.9700 \\
\hline $\mathrm{C}(26)-\mathrm{H}(26 \mathrm{~B})$ & 0.9700 \\
\hline$C(27)-C(28)$ & $1.386(13)$ \\
\hline$C(27)-C(32)$ & $1.388(13)$ \\
\hline$C(28)-C(29)$ & $1.408(13)$ \\
\hline $\mathrm{C}(28)-\mathrm{H}(28 \mathrm{~A})$ & 0.9300 \\
\hline$C(29)-C(30)$ & $1.368(15)$ \\
\hline $\mathrm{C}(29)-\mathrm{H}(29 \mathrm{~A})$ & 0.9300 \\
\hline$C(30)-C(31)$ & $1.341(15)$ \\
\hline $\mathrm{C}(30)-\mathrm{H}(30 \mathrm{~A})$ & 0.9300 \\
\hline$C(31)-C(32)$ & $1.409(14)$ \\
\hline $\mathrm{C}(31)-\mathrm{H}(31 \mathrm{~A})$ & 0.9300 \\
\hline $\mathrm{C}(32)-\mathrm{H}(32 \mathrm{~A})$ & 0.9300 \\
\hline $\mathrm{N}(16)-\mathrm{Ti}(1)-\mathrm{N}(1)$ & $171.0(3)$ \\
\hline $\mathrm{N}(16)-\mathrm{Ti}(1)-\mathrm{C}(26)$ & $85.8(3)$ \\
\hline $\mathrm{N}(1)-\mathrm{Ti}(1)-\mathrm{C}(26)$ & $100.5(3)$ \\
\hline $\mathrm{N}(16)-\mathrm{Ti}(1)-\mathrm{C}(19)$ & $98.2(3)$ \\
\hline $\mathrm{N}(1)-\mathrm{Ti}(1)-\mathrm{C}(19)$ & $86.9(3)$ \\
\hline$C(26)-T i(1)-C(19)$ & $101.0(3)$ \\
\hline $\mathrm{N}(16)-\mathrm{Ti}(1)-\mathrm{N}(10)$ & $76.3(3)$ \\
\hline $\mathrm{N}(1)-\mathrm{Ti}(1)-\mathrm{N}(10)$ & $96.0(3)$ \\
\hline $\mathrm{C}(26)-\mathrm{Ti}(1)-\mathrm{N}(10)$ & $157.9(4)$ \\
\hline $\mathrm{C}(19)-\mathrm{Ti}(1)-\mathrm{N}(10)$ & $94.3(4)$ \\
\hline $\mathrm{N}(16)-\mathrm{Ti}(1)-\mathrm{N}(7)$ & $98.4(3)$ \\
\hline $\mathrm{N}(1)-\mathrm{Ti}(1)-\mathrm{N}(7)$ & $75.1(3)$ \\
\hline $\mathrm{C}(26)-\mathrm{Ti}(1)-\mathrm{N}(7)$ & $92.7(3)$ \\
\hline $\mathrm{C}(19)-\mathrm{Ti}(1)-\mathrm{N}(7)$ & $159.1(3)$ \\
\hline $\mathrm{N}(10)-\mathrm{Ti}(1)-\mathrm{N}(7)$ & $77.5(3)$ \\
\hline$C(2)-N(1)-C(5)$ & $102.2(8)$ \\
\hline $\mathrm{C}(2)-\mathrm{N}(1)-\mathrm{Ti}(1)$ & $135.3(7)$ \\
\hline $\mathrm{C}(5)-\mathrm{N}(1)-\mathrm{Ti}(1)$ & $122.5(6)$ \\
\hline$N(1)-C(2)-C(3)$ & $108.3(9)$ \\
\hline $\mathrm{N}(1)-\mathrm{C}(2)-\mathrm{H}(2 \mathrm{~A})$ & 110.0 \\
\hline $\mathrm{C}(3)-\mathrm{C}(2)-\mathrm{H}(2 \mathrm{~A})$ & 110.0 \\
\hline $\mathrm{N}(1)-\mathrm{C}(2)-\mathrm{H}(2 \mathrm{~B})$ & 110.0 \\
\hline $\mathrm{C}(3)-\mathrm{C}(2)-\mathrm{H}(2 \mathrm{~B})$ & 110.0 \\
\hline $\mathrm{H}(2 \mathrm{~A})-\mathrm{C}(2)-\mathrm{H}(2 \mathrm{~B})$ & 108.4 \\
\hline$C(4)-C(3)-C(2)$ & $105.8(10)$ \\
\hline$C(4)-C(3)-H(3 A)$ & 110.6 \\
\hline$C(2)-C(3)-H(3 A)$ & 110.6 \\
\hline$C(4)-C(3)-H(3 B)$ & 110.6 \\
\hline$C(2)-C(3)-H(3 B)$ & 110.6 \\
\hline
\end{tabular}




\begin{tabular}{|c|c|}
\hline $\mathrm{H}(3 \mathrm{~A})-\mathrm{C}(3)-\mathrm{H}(3 \mathrm{~B})$ & 108.7 \\
\hline$C(3)-C(4)-C(5)$ & $102.7(10)$ \\
\hline $\mathrm{C}(3)-\mathrm{C}(4)-\mathrm{H}(4 \mathrm{~A})$ & 111.2 \\
\hline$C(5)-C(4)-H(4 A)$ & 111.2 \\
\hline$C(3)-C(4)-H(4 B)$ & 111.2 \\
\hline$C(5)-C(4)-H(4 B)$ & 111.2 \\
\hline $\mathrm{H}(4 \mathrm{~A})-\mathrm{C}(4)-\mathrm{H}(4 \mathrm{~B})$ & 109.1 \\
\hline$C(6)-C(5)-N(1)$ & $109.0(8)$ \\
\hline$C(6)-C(5)-C(4)$ & $114.9(11)$ \\
\hline$N(1)-C(5)-C(4)$ & $104.2(9)$ \\
\hline$C(6)-C(5)-H(5)$ & 109.5 \\
\hline $\mathrm{N}(1)-\mathrm{C}(5)-\mathrm{H}(5)$ & 109.5 \\
\hline$C(4)-C(5)-H(5)$ & 109.5 \\
\hline$C(5)-C(6)-N(7)$ & $109.8(10)$ \\
\hline$C(5)-C(6)-H(6 A)$ & 109.7 \\
\hline$N(7)-C(6)-H(6 A)$ & 109.7 \\
\hline $\mathrm{C}(5)-\mathrm{C}(6)-\mathrm{H}(6 \mathrm{~B})$ & 109.7 \\
\hline $\mathrm{N}(7)-\mathrm{C}(6)-\mathrm{H}(6 \mathrm{~B})$ & 109.7 \\
\hline $\mathrm{H}(6 \mathrm{~A})-\mathrm{C}(6)-\mathrm{H}(6 \mathrm{~B})$ & 108.2 \\
\hline$C(6)-N(7)-C(17)$ & $115.4(10)$ \\
\hline$C(6)-N(7)-C(8)$ & $110.9(9)$ \\
\hline $\mathrm{C}(17)-\mathrm{N}(7)-\mathrm{C}(8)$ & $108.3(8)$ \\
\hline $\mathrm{C}(6)-\mathrm{N}(7)-\mathrm{Ti}(1)$ & $104.3(6)$ \\
\hline $\mathrm{C}(17)-\mathrm{N}(7)-\mathrm{Ti}(1)$ & $111.1(6)$ \\
\hline $\mathrm{C}(8)-\mathrm{N}(7)-\mathrm{Ti}(1)$ & $106.5(6)$ \\
\hline$C(9)-C(8)-N(7)$ & $111.8(10)$ \\
\hline $\mathrm{C}(9)-\mathrm{C}(8)-\mathrm{H}(8 \mathrm{~A})$ & 109.3 \\
\hline $\mathrm{N}(7)-\mathrm{C}(8)-\mathrm{H}(8 \mathrm{~A})$ & 109.3 \\
\hline $\mathrm{C}(9)-\mathrm{C}(8)-\mathrm{H}(8 \mathrm{~B})$ & 109.3 \\
\hline $\mathrm{N}(7)-\mathrm{C}(8)-\mathrm{H}(8 \mathrm{~B})$ & 109.3 \\
\hline $\mathrm{H}(8 \mathrm{~A})-\mathrm{C}(8)-\mathrm{H}(8 \mathrm{~B})$ & 107.9 \\
\hline$C(8)-C(9)-N(10)$ & $109.9(10)$ \\
\hline $\mathrm{C}(8)-\mathrm{C}(9)-\mathrm{H}(9 \mathrm{~A})$ & 109.7 \\
\hline $\mathrm{N}(10)-\mathrm{C}(9)-\mathrm{H}(9 \mathrm{~A})$ & 109.7 \\
\hline $\mathrm{C}(8)-\mathrm{C}(9)-\mathrm{H}(9 \mathrm{~B})$ & 109.7 \\
\hline $\mathrm{N}(10)-\mathrm{C}(9)-\mathrm{H}(9 \mathrm{~B})$ & 109.7 \\
\hline $\mathrm{H}(9 \mathrm{~A})-\mathrm{C}(9)-\mathrm{H}(9 \mathrm{~B})$ & 108.2 \\
\hline $\mathrm{C}(9)-\mathrm{N}(10)-\mathrm{C}(11)$ & $110.4(9)$ \\
\hline$C(9)-N(10)-C(18)$ & $110.8(8)$ \\
\hline$C(11)-N(10)-C(18)$ & $108.8(10)$ \\
\hline $\mathrm{C}(9)-\mathrm{N}(10)-\mathrm{Ti}(1)$ & $109.7(7)$ \\
\hline $\mathrm{C}(11)-\mathrm{N}(10)-\mathrm{Ti}(1)$ & $104.7(6)$ \\
\hline $\mathrm{C}(18)-\mathrm{N}(10)-\mathrm{Ti}(1)$ & $112.3(6)$ \\
\hline$N(10)-C(11)-C(12)$ & $107.3(11)$ \\
\hline $\mathrm{N}(10)-\mathrm{C}(11)-\mathrm{H}(11 \mathrm{~A})$ & 110.2 \\
\hline $\mathrm{C}(12)-\mathrm{C}(11)-\mathrm{H}(11 \mathrm{~A})$ & 110.2 \\
\hline $\mathrm{N}(10)-\mathrm{C}(11)-\mathrm{H}(11 \mathrm{~B})$ & 110.2 \\
\hline $\mathrm{C}(12)-\mathrm{C}(11)-\mathrm{H}(11 \mathrm{~B})$ & 110.2 \\
\hline $\mathrm{H}(11 \mathrm{~A})-\mathrm{C}(11)-\mathrm{H}(11 \mathrm{~B})$ & 108.5 \\
\hline$N(16)-C(12)-C(13)$ & $106.3(9)$ \\
\hline $\mathrm{N}(16)-\mathrm{C}(12)-\mathrm{C}(11)$ & $108.1(9)$ \\
\hline$C(13)-C(12)-C(11)$ & $116.2(13)$ \\
\hline $\mathrm{N}(16)-\mathrm{C}(12)-\mathrm{H}(12)$ & 108.7 \\
\hline $\mathrm{C}(13)-\mathrm{C}(12)-\mathrm{H}(12)$ & 108.7 \\
\hline $\mathrm{C}(11)-\mathrm{C}(12)-\mathrm{H}(12)$ & 108.7 \\
\hline$C(14)-C(13)-C(12)$ & $101.4(11)$ \\
\hline $\mathrm{C}(14)-\mathrm{C}(13)-\mathrm{H}(13 \mathrm{~A})$ & 111.5 \\
\hline
\end{tabular}




\begin{tabular}{|c|c|}
\hline $\mathrm{C}(12)-\mathrm{C}(13)-\mathrm{H}(13 \mathrm{~A})$ & 111.5 \\
\hline $\mathrm{C}(14)-\mathrm{C}(13)-\mathrm{H}(13 \mathrm{~B})$ & 111.5 \\
\hline $\mathrm{C}(12)-\mathrm{C}(13)-\mathrm{H}(13 \mathrm{~B})$ & 111.5 \\
\hline $\mathrm{H}(13 \mathrm{~A})-\mathrm{C}(13)-\mathrm{H}(13 \mathrm{~B})$ & 109.3 \\
\hline$C(15)-C(14)-C(13)$ & $105.0(9)$ \\
\hline $\mathrm{C}(15)-\mathrm{C}(14)-\mathrm{H}(14 \mathrm{~A})$ & 110.7 \\
\hline $\mathrm{C}(13)-\mathrm{C}(14)-\mathrm{H}(14 \mathrm{~A})$ & 110.7 \\
\hline $\mathrm{C}(15)-\mathrm{C}(14)-\mathrm{H}(14 \mathrm{~B})$ & 110.7 \\
\hline $\mathrm{C}(13)-\mathrm{C}(14)-\mathrm{H}(14 \mathrm{~B})$ & 110.7 \\
\hline $\mathrm{H}(14 \mathrm{~A})-\mathrm{C}(14)-\mathrm{H}(14 \mathrm{~B})$ & 108.8 \\
\hline $\mathrm{N}(16)-\mathrm{C}(15)-\mathrm{C}(14)$ & $109.2(9)$ \\
\hline $\mathrm{N}(16)-\mathrm{C}(15)-\mathrm{H}(15 \mathrm{~A})$ & 109.8 \\
\hline $\mathrm{C}(14)-\mathrm{C}(15)-\mathrm{H}(15 \mathrm{~A})$ & 109.8 \\
\hline $\mathrm{N}(16)-\mathrm{C}(15)-\mathrm{H}(15 \mathrm{~B})$ & 109.8 \\
\hline $\mathrm{C}(14)-\mathrm{C}(15)-\mathrm{H}(15 \mathrm{~B})$ & 109.8 \\
\hline $\mathrm{H}(15 \mathrm{~A})-\mathrm{C}(15)-\mathrm{H}(15 \mathrm{~B})$ & 108.3 \\
\hline$C(12)-N(16)-C(15)$ & $102.3(9)$ \\
\hline $\mathrm{C}(12)-\mathrm{N}(16)-\mathrm{Ti}(1)$ & $122.9(7)$ \\
\hline $\mathrm{C}(15)-\mathrm{N}(16)-\mathrm{Ti}(1)$ & $134.6(7)$ \\
\hline $\mathrm{N}(7)-\mathrm{C}(17)-\mathrm{H}(17 \mathrm{~A})$ & 109.5 \\
\hline $\mathrm{N}(7)-\mathrm{C}(17)-\mathrm{H}(17 \mathrm{~B})$ & 109.5 \\
\hline $\mathrm{H}(17 \mathrm{~A})-\mathrm{C}(17)-\mathrm{H}(17 \mathrm{~B})$ & 109.5 \\
\hline $\mathrm{N}(7)-\mathrm{C}(17)-\mathrm{H}(17 \mathrm{C})$ & 109.5 \\
\hline $\mathrm{H}(17 \mathrm{~A})-\mathrm{C}(17)-\mathrm{H}(17 \mathrm{C})$ & 109.5 \\
\hline $\mathrm{H}(17 \mathrm{~B})-\mathrm{C}(17)-\mathrm{H}(17 \mathrm{C})$ & 109.5 \\
\hline $\mathrm{N}(10)-\mathrm{C}(18)-\mathrm{H}(18 \mathrm{~A})$ & 109.5 \\
\hline $\mathrm{N}(10)-\mathrm{C}(18)-\mathrm{H}(18 \mathrm{~B})$ & 109.5 \\
\hline $\mathrm{H}(18 \mathrm{~A})-\mathrm{C}(18)-\mathrm{H}(18 \mathrm{~B})$ & 109.5 \\
\hline $\mathrm{N}(10)-\mathrm{C}(18)-\mathrm{H}(18 \mathrm{C})$ & 109.5 \\
\hline $\mathrm{H}(18 \mathrm{~A})-\mathrm{C}(18)-\mathrm{H}(18 \mathrm{C})$ & 109.5 \\
\hline $\mathrm{H}(18 \mathrm{~B})-\mathrm{C}(18)-\mathrm{H}(18 \mathrm{C})$ & 109.5 \\
\hline$C(20)-C(19)-T i(1)$ & $123.3(6)$ \\
\hline $\mathrm{C}(20)-\mathrm{C}(19)-\mathrm{H}(19 \mathrm{~A})$ & 106.5 \\
\hline $\mathrm{Ti}(1)-\mathrm{C}(19)-\mathrm{H}(19 \mathrm{~A})$ & 106.5 \\
\hline $\mathrm{C}(20)-\mathrm{C}(19)-\mathrm{H}(19 \mathrm{~B})$ & 106.5 \\
\hline $\mathrm{Ti}(1)-\mathrm{C}(19)-\mathrm{H}(19 \mathrm{~B})$ & 106.5 \\
\hline $\mathrm{H}(19 \mathrm{~A})-\mathrm{C}(19)-\mathrm{H}(19 \mathrm{~B})$ & 106.5 \\
\hline$C(25)-C(20)-C(21)$ & $117.2(10)$ \\
\hline$C(25)-C(20)-C(19)$ & $121.4(9)$ \\
\hline$C(21)-C(20)-C(19)$ & $121.4(10)$ \\
\hline$C(22)-C(21)-C(20)$ & $123.1(11)$ \\
\hline $\mathrm{C}(22)-\mathrm{C}(21)-\mathrm{H}(21 \mathrm{~A})$ & 118.4 \\
\hline $\mathrm{C}(20)-\mathrm{C}(21)-\mathrm{H}(21 \mathrm{~A})$ & 118.4 \\
\hline$C(21)-C(22)-C(23)$ & $117.8(14)$ \\
\hline $\mathrm{C}(21)-\mathrm{C}(22)-\mathrm{H}(22 \mathrm{~A})$ & 121.1 \\
\hline $\mathrm{C}(23)-\mathrm{C}(22)-\mathrm{H}(22 \mathrm{~A})$ & 121.1 \\
\hline$C(24)-C(23)-C(22)$ & $119.7(14)$ \\
\hline $\mathrm{C}(24)-\mathrm{C}(23)-\mathrm{H}(23 \mathrm{~A})$ & 120.2 \\
\hline $\mathrm{C}(22)-\mathrm{C}(23)-\mathrm{H}(23 \mathrm{~A})$ & 120.2 \\
\hline$C(23)-C(24)-C(25)$ & $122.6(13)$ \\
\hline $\mathrm{C}(23)-\mathrm{C}(24)-\mathrm{H}(24 \mathrm{~A})$ & 118.7 \\
\hline $\mathrm{C}(25)-\mathrm{C}(24)-\mathrm{H}(24 \mathrm{~A})$ & 118.7 \\
\hline$C(20)-C(25)-C(24)$ & $119.5(11)$ \\
\hline $\mathrm{C}(20)-\mathrm{C}(25)-\mathrm{H}(25 \mathrm{~A})$ & 120.2 \\
\hline $\mathrm{C}(24)-\mathrm{C}(25)-\mathrm{H}(25 \mathrm{~A})$ & 120.2 \\
\hline $\mathrm{C}(27)-\mathrm{C}(26)-\mathrm{Ti}(1)$ & $123.8(6)$ \\
\hline $\mathrm{C}(27)-\mathrm{C}(26)-\mathrm{H}(26 \mathrm{~A})$ & 106.4 \\
\hline
\end{tabular}




\begin{tabular}{ll}
$\mathrm{Ti}(1)-\mathrm{C}(26)-\mathrm{H}(26 \mathrm{~A})$ & 106.4 \\
$\mathrm{C}(27)-\mathrm{C}(26)-\mathrm{H}(26 \mathrm{~B})$ & 106.4 \\
$\mathrm{Ti}(1)-\mathrm{C}(26)-\mathrm{H}(26 \mathrm{~B})$ & 106.4 \\
$\mathrm{H}(26 \mathrm{~A})-\mathrm{C}(26)-\mathrm{H}(26 \mathrm{~B})$ & 106.4 \\
$\mathrm{C}(28)-\mathrm{C}(27)-\mathrm{C}(32)$ & $113.1(11)$ \\
$\mathrm{C}(28)-\mathrm{C}(27)-\mathrm{C}(26)$ & $122.3(10)$ \\
$\mathrm{C}(32)-\mathrm{C}(27)-\mathrm{C}(26)$ & $124.6(10)$ \\
$\mathrm{C}(27)-\mathrm{C}(28)-\mathrm{C}(29)$ & $123.2(11)$ \\
$\mathrm{C}(27)-\mathrm{C}(28)-\mathrm{H}(28 \mathrm{~A})$ & 118.4 \\
$\mathrm{C}(29)-\mathrm{C}(28)-\mathrm{H}(28 \mathrm{~A})$ & 118.4 \\
$\mathrm{C}(30)-\mathrm{C}(29)-\mathrm{C}(28)$ & $120.3(12)$ \\
$\mathrm{C}(30)-\mathrm{C}(29)-\mathrm{H}(29 \mathrm{~A})$ & 119.9 \\
$\mathrm{C}(28)-\mathrm{C}(29)-\mathrm{H}(29 \mathrm{~A})$ & 119.9 \\
$\mathrm{C}(31)-\mathrm{C}(30)-\mathrm{C}(29)$ & $119.4(14)$ \\
$\mathrm{C}(31)-\mathrm{C}(30)-\mathrm{H}(30 \mathrm{~A})$ & 120.3 \\
$\mathrm{C}(29)-\mathrm{C}(30)-\mathrm{H}(30 \mathrm{~A})$ & 120.3 \\
$\mathrm{C}(30)-\mathrm{C}(31)-\mathrm{C}(32)$ & $119.3(12)$ \\
$\mathrm{C}(30)-\mathrm{C}(31)-\mathrm{H}(31 \mathrm{~A})$ & 120.3 \\
$\mathrm{C}(32)-\mathrm{C}(31)-\mathrm{H}(31 \mathrm{~A})$ & 120.3 \\
$\mathrm{C}(27)-\mathrm{C}(32)-\mathrm{C}(31)$ & $124.7(11)$ \\
$\mathrm{C}(27)-\mathrm{C}(32)-\mathrm{H}(32 \mathrm{~A})$ & 117.7 \\
$\mathrm{C}(31)-\mathrm{C}(32)-\mathrm{H}(32 \mathrm{~A})$ & 117.7 \\
\hline
\end{tabular}

Symmetry transformations used to generate equivalent atoms: 
Table 5. Anisotropic displacement parameters $\left(A^{\wedge} 2 \times 10^{\wedge} 3\right)$ for $C_{2}-6$. The anisotropic displacement factor exponent takes the form:

$-2 \mathrm{pi}^{\wedge} 2\left[\mathrm{~h}^{\wedge} 2 \mathrm{a}^{\star \wedge} 2 \mathrm{U} 11+\ldots+2 \mathrm{hk} \mathrm{a}\right.$ * $\left.\mathrm{b} * \mathrm{U} 12\right]$

\begin{tabular}{|c|c|c|c|c|c|c|}
\hline & U11 & $\mathrm{U} 22$ & U3 3 & $\mathrm{U} 23$ & U13 & $\mathrm{U} 12$ \\
\hline $\operatorname{Ti}(1)$ & $34(1)$ & $30(1)$ & $69(1)$ & $11(1)$ & $10(1)$ & $19(1)$ \\
\hline$N(1)$ & $39(3)$ & $34(4)$ & $66(3)$ & $13(3)$ & $1(3)$ & $12(3)$ \\
\hline$C(2)$ & $51(3)$ & $62(7)$ & $69(5)$ & $20(5)$ & $14(3)$ & $15(5)$ \\
\hline$C(3)$ & $101(5)$ & $110(10)$ & $67(5)$ & $24(8)$ & $21(5)$ & $36(7)$ \\
\hline$C(4)$ & $117(6)$ & $94(9)$ & $67(4)$ & $26(6)$ & $-16(4)$ & $47(7)$ \\
\hline$C(5)$ & $70(4)$ & $57(7)$ & $93(5)$ & $30(6)$ & $-13(4)$ & $29(4)$ \\
\hline$C(6)$ & $58(3)$ & $84(7)$ & $127(4)$ & $17(6)$ & $-36(4)$ & $25(5)$ \\
\hline$N(7)$ & $41(3)$ & $63(4)$ & $147(4)$ & $35(4)$ & $2(3)$ & $34(3)$ \\
\hline$C(8)$ & $83(8)$ & $86(5)$ & $200(9)$ & $52(7)$ & $32(7)$ & $72(6)$ \\
\hline C (9) & $104(5)$ & $66(7)$ & $190(8)$ & $46(6)$ & $73(7)$ & $69(5)$ \\
\hline $\mathrm{N}(10)$ & $89(5)$ & $42(3)$ & $126(4)$ & $10(3)$ & $46(4)$ & $39(4)$ \\
\hline$C(11)$ & $136(10)$ & $63(4)$ & $116(4)$ & $-2(4)$ & $57(6)$ & $59(7)$ \\
\hline$C(12)$ & $103(10)$ & $75(4)$ & $104(5)$ & $14(5)$ & $59(7)$ & $52(6)$ \\
\hline C (13) & $149(12)$ & $101(5)$ & $80(4)$ & $10(4)$ & $65(8)$ & $71(8)$ \\
\hline$C(14)$ & $154(11)$ & $93(5)$ & $84(5)$ & $30(5)$ & $60(10)$ & $61(7)$ \\
\hline C (15) & $81(7)$ & $52(4)$ & $84(5)$ & $25(3)$ & $34(6)$ & $20(5)$ \\
\hline $\mathrm{N}(16)$ & $56(5)$ & $45(3)$ & $81(3)$ & $15(3)$ & $32(3)$ & $25(4)$ \\
\hline C (17) & $33(3)$ & $91(5)$ & $203(11)$ & $35(7)$ & $-2(6)$ & $17(5)$ \\
\hline$C(18)$ & $112(6)$ & $32(3)$ & $150(10)$ & $10(5)$ & $35(8)$ & $35(4)$ \\
\hline C (19) & $42(5)$ & $41(6)$ & $49(7)$ & $-9(5)$ & $-6(5)$ & $21(5)$ \\
\hline$C(20)$ & $35(6)$ & $35(6)$ & $33(7)$ & $-2(5)$ & $-6(5)$ & $4(5)$ \\
\hline C (21) & $97(10)$ & $51(7)$ & $79(9)$ & $-16(8)$ & $-22(8)$ & $35(7)$ \\
\hline$C(22)$ & $133(13)$ & $116(12)$ & $71(10)$ & $-23(10)$ & $-48(9)$ & $64(10)$ \\
\hline C (23) & $99(10)$ & $125(13)$ & $88(12)$ & $40(12)$ & $-7(9)$ & $53(11)$ \\
\hline C (24) & $68(9)$ & $70(10)$ & $117(14)$ & $27(9)$ & $-11(8)$ & $26(7)$ \\
\hline C (25) & $67(7)$ & $56(8)$ & $85(11)$ & $-5(7)$ & $-34(8)$ & $41(6)$ \\
\hline$C(26)$ & $60(6)$ & $22(5)$ & $69(8)$ & $-3(5)$ & $-14(5)$ & $24(5)$ \\
\hline C (27) & $33(6)$ & $38(6)$ & $72(9)$ & $-4(6)$ & $-29(6)$ & $15(5)$ \\
\hline$C(28)$ & $81(9)$ & $52(8)$ & $84(10)$ & $-21(7)$ & $-17(8)$ & $39(8)$ \\
\hline C (29) & $75(9)$ & $71(8)$ & $89(11)$ & $-9(7)$ & $1(9)$ & $32(8)$ \\
\hline C (30) & $132(13)$ & $75(9)$ & $84(11)$ & $-18(9)$ & $-9(12)$ & $53(11)$ \\
\hline$C(31)$ & $85(10)$ & $76(9)$ & $72(11)$ & $-13(8)$ & $-44(9)$ & $22(8)$ \\
\hline C (32) & $64(7)$ & $58(7)$ & $61(9)$ & $-7(7)$ & $-20(7)$ & $23(7)$ \\
\hline
\end{tabular}


Table 6. Hydrogen coordinates $\left(\mathrm{x}_{\left.10^{\wedge} 4\right)}\right)$ and isotropic displacement parameters $\left(A^{\wedge} 2 \times 10^{\wedge} 3\right)$ for $C_{2}-6$.

\begin{tabular}{|c|c|c|c|c|}
\hline & $\mathrm{x}$ & $\mathrm{Y}$ & z & $\mathrm{U}(\mathrm{eq})$ \\
\hline $\mathrm{H}(2 \mathrm{~A})$ & 12947 & 6889 & 9269 & 80 \\
\hline $\mathrm{H}(2 \mathrm{~B})$ & 12689 & 8603 & 9246 & 80 \\
\hline$H(3 A)$ & 12635 & 8247 & 8917 & 120 \\
\hline $\mathrm{H}(3 \mathrm{~B})$ & 12605 & 6373 & 8942 & 120 \\
\hline $\mathrm{H}(4 \mathrm{~A})$ & 9596 & 4765 & 8914 & 114 \\
\hline $\mathrm{H}(4 \mathrm{~B})$ & 9761 & 6566 & 8819 & 114 \\
\hline $\mathrm{H}(5)$ & 9284 & 7535 & 9126 & 89 \\
\hline $\mathrm{H}(6 \mathrm{~A})$ & 7083 & 3724 & 9127 & 113 \\
\hline $\mathrm{H}(6 \mathrm{~B})$ & 6462 & 5167 & 9085 & 113 \\
\hline $\mathrm{H}(8 \mathrm{~A})$ & 7728 & 7654 & 9362 & 132 \\
\hline $\mathrm{H}(8 \mathrm{~B})$ & 5637 & 6507 & 9415 & 132 \\
\hline $\mathrm{H}(9 \mathrm{~A})$ & 6319 & 6133 & 9746 & 130 \\
\hline $\mathrm{H}(9 \mathrm{~B})$ & 7297 & 8232 & 9695 & 130 \\
\hline $\mathrm{H}(11 \mathrm{~A})$ & 8866 & 7738 & 10013 & 121 \\
\hline $\mathrm{H}(11 \mathrm{~B})$ & 10450 & 7312 & 9969 & 121 \\
\hline $\mathrm{H}(12)$ & 6641 & 4733 & 9982 & 109 \\
\hline $\mathrm{H}(13 \mathrm{~A})$ & 9546 & 4694 & 10199 & 127 \\
\hline $\mathrm{H}(13 \mathrm{~B})$ & 7734 & 4504 & 10294 & 127 \\
\hline $\mathrm{H}(14 \mathrm{~A})$ & 7901 & 1606 & 10195 & 133 \\
\hline $\mathrm{H}(14 \mathrm{~B})$ & 6019 & 1581 & 10189 & 133 \\
\hline $\mathrm{H}(15 \mathrm{~A})$ & 7898 & 1414 & 9863 & 94 \\
\hline $\mathrm{H}(15 \mathrm{~B})$ & 5966 & 1266 & 9858 & 94 \\
\hline $\mathrm{H}(17 \mathrm{~A})$ & 4039 & 3284 & 9379 & 173 \\
\hline $\mathrm{H}(17 \mathrm{~B})$ & 4865 & 3367 & 9595 & 173 \\
\hline $\mathrm{H}(17 \mathrm{C})$ & 5026 & 2164 & 9423 & 173 \\
\hline $\mathrm{H}(18 \mathrm{~A})$ & 10574 & 10041 & 9736 & 149 \\
\hline $\mathrm{H}(18 \mathrm{~B})$ & 10480 & 9361 & 9512 & 149 \\
\hline $\mathrm{H}(18 \mathrm{C})$ & 11757 & 9167 & 9671 & 149 \\
\hline $\mathrm{H}(19 \mathrm{~A})$ & 12667 & 5511 & 9558 & 52 \\
\hline $\mathrm{H}(19 \mathrm{~B})$ & 12762 & 7277 & 9643 & 52 \\
\hline $\mathrm{H}(21 \mathrm{~A})$ & 13856 & 8143 & 9969 & 91 \\
\hline $\mathrm{H}(22 \mathrm{~A})$ & 14549 & 7584 & 10291 & 127 \\
\hline $\mathrm{H}(23 \mathrm{~A})$ & 13774 & 4584 & 10376 & 126 \\
\hline $\mathrm{H}(24 \mathrm{~A})$ & 12237 & 2316 & 10153 & 107 \\
\hline $\mathrm{H}(25 \mathrm{~A})$ & 11563 & 2863 & 9826 & 77 \\
\hline $\mathrm{H}(26 \mathrm{~A})$ & 9059 & 1730 & 9559 & 59 \\
\hline $\mathrm{H}(26 \mathrm{~B})$ & 7167 & 1429 & 9490 & 59 \\
\hline $\mathrm{H}(28 \mathrm{~A})$ & 11532 & 3031 & 9257 & 83 \\
\hline $\mathrm{H}(29 \mathrm{~A})$ & 12050 & 2280 & 8934 & 97 \\
\hline $\mathrm{H}(30 \mathrm{~A})$ & 9634 & 340 & 8729 & 116 \\
\hline $\mathrm{H}(31 \mathrm{~A})$ & 6714 & -700 & 8840 & 103 \\
\hline $\mathrm{H}(32 \mathrm{~A})$ & 6206 & 41 & 9163 & 77 \\
\hline
\end{tabular}

